\title{
Inhaled Gases for Neuroprotection of Neonates: A Review
}

\begin{abstract}
Youness Tolaymat ${ }^{1 *}$, Sylvain Doré ${ }^{2}$, Hudson W. Griffin ${ }^{3}$, Susana Shih ${ }^{3}$, Mary E. Edwards ${ }^{4}$ and Michael D. Weiss ${ }^{1}$

${ }^{1}$ Department of Pediatrics, University of Florida, Gainesville, FL, United States, ${ }^{2}$ Departments of Neurology, Psychiatry, Pharmaceuticals and Neuroscience, University of Florida, Gainesville, FL, United States, ${ }^{3}$ Department of Anesthesiology, University of Florida, Gainesville, FL, United States, ${ }^{4}$ Health Science Center Libraries, University of Florida, Gainesville, FL, United States
\end{abstract}

Importance: Hypoxic-ischemic encephalopathy $(\mathrm{HIE})$ is a significant cause of morbidity and mortality in neonates. The incidence of $\mathrm{HIE}$ is 1-8 per 1,000 live births in developed countries. Whole-body hypothermia reduces the risk of disability or death, but 7 infants needed to be treated to prevent death or major neurodevelopmental disability. Inhalational gases may be promising synergistic agents due to their rapid onset and easy titratability.

Objective: To review current data on different inhaled gases with neuroprotective properties that may serve as adjunct therapies to hypothermia.

Evidence review: Literature review was performed using the PubMed database, google scholar, and ClinicalTrials.Gov. Results focused on articles published from January 1, 2005, through December 31, 2017. Articles published earlier than 2005 were included when appropriate for historical perspective. Our review emphasized preclinical and clinical studies relevant to the use of inhaled agents for neuroprotection.

Findings: Based on the relevance to our topic, 111 articles were selected pertaining to the incidence of HIE, pathophysiology of HIE, therapeutic hypothermia, and emerging therapies for hypoxic-ischemic encephalopathy in preclinical and clinical settings. Supplemental tables summarizes highly relevant 49 publications that were included in this review. The selected publications emphasize the emergence of promising inhaled gases that may improve neurologic survival and alleviate neurodevelopmental disability when combined with therapeutic hypothermia in the future.

Conclusions: Many inhaled agents have neuroprotective properties and could serve as an adjunct therapy to whole-body hypothermia. Inhaled agents are ideal due to their easy administration, titrability, and rapid onset and offset.

\section{Keywords: HIE, hypoxia-ischemia, inhaled gases, neuroprotection, neonatal}

\section{INTRODUCTION}

Hypoxic-ischemic encephalopathy (HIE) is a significant cause of morbidity and mortality in neonates. The incidence of HIE ranges from 1 to 8 per 1,000 live births in developed countries as high as 26 per 1,000 live births in underdeveloped countries (1).

Whole-body hypothermia reduces the risk of death or disability in infants with moderate or severe HIE (2-4). A metanalysis of 11 cooling trials in neonates with moderate to severe HIE demonstrated that to save 1 infant from either death or major neurodevelopmental disability, 7 infants must be cooled (5). In 2014, a multicenter randomized 
clinical trial evaluated increasing the depth of hypothermia to $32.0^{\circ} \mathrm{C}$ and the duration to $120 \mathrm{~h}$, a change from original trials and the current clinical regimen of cooling to $33.5^{\circ} \mathrm{C}$ for $72 \mathrm{~h}$. In this study infants were randomized to four hypothermia groups: $33.5^{\circ} \mathrm{C}$ for $72 \mathrm{~h}, 33.5^{\circ} \mathrm{C}$ for $120 \mathrm{~h}, 32^{\circ} \mathrm{C}$ for $72 \mathrm{~h}$, or $32^{\circ} \mathrm{C}$ for $120 \mathrm{~h}$. However, the study was terminated early due to concern of increased mortality that was higher in infants who underwent longer and deeper cooling (6). Of the children who were enrolled and were followed-up at 18-22 months of age, the primary outcome of death or severe neurodevelopmental disability at $18-22$ months was $29.3 \%$ at $33.5^{\circ} \mathrm{C}$ for $72 \mathrm{~h}, 34.5 \%$ at $32.0^{\circ} \mathrm{C}$ for $72 \mathrm{~h}, 34.4 \%$ at $33.5^{\circ} \mathrm{C}$ for $120 \mathrm{~h}$, and $28.2 \%$ at $32.0^{\circ} \mathrm{C}$ for $120 \mathrm{~h}$ (7). Therefore, we should strive for additional adjunct therapies to hypothermia that improve neurodevelopmental outcomes. Inhalational gases may serve as an ideal synergistic agent with hypothermia for neuroprotection due to their rapid onset and offset and titratability. This review will examine several promising inhalational gases that may offer synergistic neuroprotection when combined with hypothermia.

Currently, medical gases are commonly used in the medical field. Nitrous oxide and xenon are used as anesthetic agents, nitric oxide is used to treat pulmonary hypertension in neonates and adults and heliox is used in patients with upper and lower airway obstruction. The use of medical gases may be limited by cost. Nitric oxide treatment currently costs more than $\$ 100$ per $h$, while xenon can costs $\$ 300$ per h due to its rarity in atmospheric air $(8,9)$.

The use of inhaled gases as neuroprotective adjuncts with hypothermia warrants further evaluation of gas stability and effect on bioavailability and pharmacokinetics. While most reviewed studies reviewed in this article looked at inhaled gases in normothermic conditions, some animal studies have evaluated the use of inhaled gases in the setting of hypothermia (10-14). Furthermore, TOBY-Xe trial assessed the use of inhaled xenon as an adjunct therapy to therapeutic hypothermia (15).

\section{PATHOPHYSIOLOGY OF HIE}

The mechanism of injury during hypoxia-ischemia (HI) provides a foundation for understanding the neuroprotective mechanisms of various inhalational gases. Oxygen and glucose are delivered to the brain in fetal life by cerebral blood flow. Cerebral blood flow helps the fetal brain maintain homeostasis and meet cellular energy demands. Acute sentinel events including placental abruption, prolapse of the umbilical cord, and uterine

\footnotetext{
Abbreviations: AMPA, Alpha-Amino-3-Hydroxy-5-Methyl-4-Isoxazole Propionic Acid; ARE, antioxidant response elements; Bcl-2, B-cell lymphoma 2; $\mathrm{CO}$, carbon monoxide; $\mathrm{CO}_{2}$, carbon dioxide; GABA receptors, $\gamma$ aminobutyric acid type A receptors; HI, hypoxia-ischemia; HIE, hypoxic-ischemic encephalopathy; HO1, heme-oxygenase 1; iNO, inhaled nitrous oxide; KA, kainite; Keap1, kelch-like ECH-associated protein 1; LDH, lactate dehydrogenase; L-NAME, $\mathrm{N}(\omega)$-nitro-L-arginine methyl ester; MCAO, middle cerebral artery occlusion; NAA, N-acetyl aspartate; NF- $\kappa \mathrm{B}$, nuclear factor- $\kappa \mathrm{B}$; NMDA, Nmethyl-D-aspartate; NO, nitric oxide; NRF2, nuclear erythroid 2-related factor 2; OGD, oxygen and glucose deprivation; pCREB, phosphorylated cAMP response element binding protein; ROS, reactive oxygen species; Tyr216-GSK-3 $\beta$, Phospho-glycogen synthase kinase-3 $\beta$ Tyr-216.
}

rupture decrease placental perfusion or disrupt the delivery of oxygen and glucose in the umbilical cord. Poor oxygenation from the placenta produces fetal hypoxia, which eventually leads to a decrease in fetal cardiac output. Subsequently, decreased fetal cardiac output reduces cerebral blood flow. Decreased cerebral perfusion sets in motion a temporal sequence of injury, which has distinct phases. In the acute phase, interruption of cerebral blood flow diminishes the delivery of oxygen and glucose to the fetal brain switching the cellular metabolism to anerobic glycolysis. Consequently, diminished ATP production leads to disturbances in intracellular sodium, water, and calcium as a result of dysfunctional transcellular transport (16). During depolarization, the excitatory amino acid glutamate, is released from presynaptic neurons. During hypoxia-ischemia there is an increased release of glutamate that leads to overstimulation of the glutamate receptors [i.e., $\alpha$-amino-3-hydroxy-5-methyl-4-isoxazole propionate (AMPA), kainite (KA), and N-methyl-D-aspartate (NMDA)] on the postsynaptic neuron. Excitotoxicity results from overstimulation of glutamate receptors. Overstimulation of the KA and AMPA receptors causes sodium and chloride influx, leading to cellular hyperosmolality. Thus, water influx results in subcellular edema, which if severe enough can cause cell lysis. Overstimulation of the NMDA receptor triggers an influx of calcium. Calcium is the predominant second messenger in the cell. When calcium is present in pathological amounts, a series of enzymes are activated that destroys the cell. Calcium also contributes to the production of free radicals such as nitric oxide (NO), Fatty acid, and prostaglandin metabolism also generate free radicals, which lead to the formation of harmful amounts of superoxide and hydrogen peroxide. This cascade of events perpetuates injury through excitotoxicity. Free fatty acid peroxidation mediated by oxygen radicals causes worsening of the cellular injury (17). The culmination of energy failure, acidosis, glutamate mediated excitotoxicity, lipid peroxidation, and NO toxicity causes necrosis and apoptosis of cells in the brain (17).

A partial recovery ensues over the 30-60 min after resuscitation following the primary phase of injury. This partial recovery leads to a latent phase of injury (18). The latent phase may persist from 1 to $6 \mathrm{~h}$ and is characterized by recovery of oxidative metabolism, inflammation, and continuation of the activated apoptotic cascades (16). A secondary deterioration follows the latent phase in neonates with moderate to severe injury. The secondary phase of injury occurs within $\sim 6-15 \mathrm{~h}$ after the injury. Cytotoxic edema, excitotoxicity, and "secondary energy failure" with nearly complete failure of mitochondrial activity characterize this second phase. Seizures typically occur in this secondary phase (19). A tertiary phase occurs over months after the acute insult and involves late cell death, remodeling of the injured brain, and astrogliosis (20).

Therapeutic hypothermia interrupts the pathophysiologic cascade of HIE during the acute phase of injury by limiting apoptosis and allowing time for recovery from oxidative stress. Proinflammatory pathway involved in the pathophysiology of HIE and characterized by production of cytokines such as IL-6, IL-8, and platelet-activating factor (PAF) are halted by $72 \mathrm{~h}$ of hypothermia $(21,22)$ Furthermore, excitotoxicity 
caused by influx of calcium is decreased by hypothermia (23). Hypothermia, in rats models of $\mathrm{HI}$, prevents injury to the mitochondria by suppressing cytochrome $\mathrm{C}$ during the acute phase of injury (24).

\section{EVIDENCE REVIEW}

We performed a literature review using the PubMed database, google scholar, and ClinicalTrials.Gov. The results focused on articles published between 2005 and 2017. Articles published prior to 2005 were incorporated when appropriate for historical perspective. Our review highlighted relevant preclinical and clinical studies that examined the use of inhaled agents for neuroprotection. Due to the overlap between adult stroke and neonatal $\mathrm{HI}$, although the pathophysiology in $\mathrm{HI}$ tends to be more global, adult stroke studies were included because the studies provide potential information on therapies that may apply to neonatal $\mathrm{HI}$ and offer potential clues as to the mechanism of action of inhaled gases in neonatal HI. Therefore, we have included information from adult animal studies because neonatal studies are limited to broaden the perspective of each gas and the use as a potential therapy in neonatal HI.

In this article, we will discuss the emerging medical gases that have been evaluated in preclinical and clinical trials for neuroprotection in neonatal hypoxia-ischemia. Although many articles exist, it is beyond the scope of this review to focus on all articles related to the presented inhalational gases and every inhalational gas in the literature. To the make the review informative but concise, we have tried to select articles that provide representative information on the potential for each gas for neuroprotection in the preclinical and clinical setting and that highlight the potential mechanism of neuroprotection. We have selected inhalational gases that have demonstrated promise for potential translational applications in neonates with HIE. The text is augmented by Tables 1, 2 that briefly describe other articles from the literature that are not covered in the text.

\section{Xenon}

Xenon is one of the most studied inhaled gases. As a noble gas, it is not reactive under normal conditions. Xenon crosses the blood-brain barrier, has a fast onset of action, is an anesthetic at atmospheric pressure, and is safe in neonates undergoing hypothermia (51). Xenon is approved as an anesthetic drug. A multicenter international study showed that inhaled $65 \%$ xenon was not inferior to inhaled sevoflurane or intravenous propofol as an anesthetic agent in coronary bypass graft surgery. Furthermore, xenon administration was associated with less troponin-I release when compared to propofol (69). It is cardioand nephro-protective and is not fetotoxic $(53,70,71)$. Xenon's neuroprotective effects are mediated by antagonizing the NMDA receptor channel $(72,73)$. Xenon also blocks AMPA, which is a glutamate receptor (74). In addition, xenon interferes with the calcium/calmodulin-activated kinase II complex (75). Although xenon has properties that make it an ideal therapeutic gas, it is expensive and scarce in atmospheric air. Xenon has no effect on heart rate and improves blood pressure during hypothermia in a preclinical piglet study (67).

\section{Preclinical Studies}

Xenon is a potential neuroprotective agent following hypoxic-ischemic injury because it transiently blocks NMDA and AMPA receptors involved with excitotoxicity (Supplementary Figure 1). Neonatal rats that experienced left carotid artery ligation for $90 \mathrm{~min}$ were treated with $3 \mathrm{~h}$ of inhaled $50 \%$ xenon. Histopathological evaluation 1 week from the time of injury showed that rats treated with xenon had improved preservation of the cortex, hippocampus, basal ganglia, and thalamus (76).

Xenon preconditioning is also preventive against ischemia-induced neuronal injury. In vitro, pre-exposure to xenon before oxygen and glucose deprivation (OGD) reduces lactate dehydrogenase (LDH) and propidium iodide staining in neuronal cell cultures. Neonatal rats undergoing HI following preconditioning with $70 \%$ xenon had smaller infarct sizes and elevated levels of phosphorylated cAMP response element-binding protein (pCREB) and B-cell lymphoma 2 (Bcl-2) (54). Bcl-2 is a prosurvival protein that decreases apoptosis and is upregulated by pCREB (77).

Sevoflurane, a volatile anesthetic that blocks $\gamma$-aminobutyric acid type A receptors (GABA), synergistically augments the neuroprotective effects of xenon. In vitro, preconditioning with a combination of xenon and sevoflurane for $2 \mathrm{~h}$ before OGD had a greater reduction of $\mathrm{LDH}$ release. Although sevoflurane alone did not reduce infarct size in a Rice-Vannucci model of neonatal hypoxic-ischemic injury, xenon alone and in combination with sevoflurane reduced infarct size and improved neuromotor function at 30 days. The xenon-sevoflurane group had a slightly improved effect (52). In another study, pregnant rats were preconditioned at day 22 of gestation with $35 \%$ xenon or $0.35 \%$ sevoflurane before sacrificing dams. After sacrifice, uterine horns were placed in $37^{\circ} \mathrm{C}$ water for $10 \mathrm{~min}$ to simulate neonatal asphyxia. Compared to the control group, xenon- and sevoflurane-preconditioned pups had lower caspase-3 levels in the hippocampus and higher cell viability at postnatal days 3 and 7. These preconditioned pups also showed improved cognition in the Morris water maze test at postnatal day 50 (56).

Neonatal rats undergoing the Rice-Vannucci model of hypoxic-ischemic injury for $90 \mathrm{~min}$ received xenon at 1 and $5 \mathrm{~h}$ after initiation of hypothermia $\left(32^{\circ} \mathrm{C}\right)$. These rats had significantly reduced infarct volume (13). A combined therapy of xenon and hypothermia was found to decrease infarct volume and improved short and long-term functional testing compared to each treatment alone $(11,12)$. However, a recent study with a larger sample size by the same group failed to replicate the results and did not show a synergistic effect (55). In another study, neonatal rats received xenon combined with hypothermia after undergoing hypoxia for $150 \mathrm{~min}$ due to common carotid ligation. These rats did not show altered hemispheric loss (14). Hypothermia augmented with xenon in asphyxiated newborn piglets had decreased markers of brain injury as measured by cerebral magnetic resonance spectroscopy, decreased nuclear DNA fragmentation using TUNEL staining and caspase 3 activity in the cortex. However, combining xenon with hypothermia was not superior to hypothermia alone (68). 
TABLE 1 | Summary of preclinical studies of inhaled gases in rodents.

\begin{tabular}{|c|c|c|c|c|c|c|}
\hline References & Gas & Age species & Procedure & Intervention & Outcomes & Behavioral \\
\hline $\begin{array}{l}\text { David et al. } \\
\text { (25) }\end{array}$ & $\mathrm{Ar}$ & $\begin{array}{l}\text { Adult male } \\
\text { rats }\end{array}$ & $\begin{array}{l}\text { a) In vitro: neuronal cell } \\
\text { slices were subjected } \\
\text { to OGD } \\
\text { b) In vivo: injection of } \\
\text { NMDA into right } \\
\text { striatum } \\
\text { c) In vivo: MCAO } \\
\text { transient ischemia for } \\
1 \mathrm{~h}\end{array}$ & $\begin{array}{l}\text { a) OGD: Reperfusion and exposure to } 25 \text {, } \\
37.5,50 \text {, or } 75 \% \text { Ar } \\
\text { b) NMDA: } 1 \mathrm{~h} \text { after injection of NMDA rats } \\
\text { were treated with either medical air } \\
\text { (control), or } 15,25,37.5,50 \text {, or } 75 \% \mathrm{Ar} \\
\text { balanced with } \mathrm{O}_{2} \text { or } \mathrm{N}_{2} \\
\text { c) } 3 \mathrm{~h} \text { post MCAO, rats were treated with } \\
\text { either medical air (control) or } 50 \% \mathrm{Ar}\end{array}$ & $\begin{array}{l}\text { a) Less LDH production in Ar group } \\
\text { b) Rats treated with } 37.5 \text { and } 50 \% \\
\text { Ar had a significant reduction of } \\
\text { neuronal death compared to } \\
\text { NMDA control animals treated } \\
\text { with medical air } \\
\text { c) In MCAO, maximal } \\
\text { neuroprotection by post-insult } \\
50 \% \text { Ar. When given } 1 \mathrm{~h} \text { after } \\
\text { reperfusion, Ar reduced cortical } \\
\text { volumes of brain damage but } \\
\text { increased subcortical brain } \\
\text { damage compared to control rats } \\
\text { treated with air }\end{array}$ & $\begin{array}{l}\text { Behavioral outcome assessed only } \\
\text { for MCAO study rats. Rats treated } \\
\text { with } 50 \% \text { Ar did not show } \\
\text { improvement of neurologic deficits } \\
\text { compared to control rats treated with } \\
\text { medical air }\end{array}$ \\
\hline $\begin{array}{l}\text { Loetscher } \\
\text { et al. (26) }\end{array}$ & $\mathrm{Ar}$ & Neonatal mice & $\begin{array}{l}\text { Hippocampal slice } \\
\text { cultures subjected to } \\
\text { either OGD or a focal } \\
\text { mechanical trauma }\end{array}$ & $\begin{array}{l}25,50 \text {, or } 74 \% \text { Ar immediately after trauma } \\
\text { or with a } 2 \text { or } 3 \mathrm{~h} \text { delay }\end{array}$ & $\begin{array}{l}\text { a) Ar is neuroprotective in both } \mathrm{O}_{2} \\
\text { deprivation and mechanical brain } \\
\text { trauma even when therapy is } \\
\text { delayed for } 3 \mathrm{~h} \\
\text { b) No difference in } \mathrm{O}_{2} \text { deprivation } \\
\text { between } \mathrm{Ar} \text { concentrations while } \\
50 \% \mathrm{Ar} \text { had best outcomes in } \\
\text { mechanical brain trauma }\end{array}$ & $\mathrm{N} / \mathrm{A}$ \\
\hline $\begin{array}{l}\text { Ryang et al. } \\
(27)\end{array}$ & $\mathrm{Ar}$ & Adult rats & MCAO $2 \mathrm{~h}$ & $\begin{array}{l}\text { Two groups: } \\
\text { a) } 50 \% \mathrm{Ar}+50 \% \mathrm{O}_{2} \text { (Ar group) } \\
\text { b) } 50 \% \mathrm{~N}_{2}+50 \% \mathrm{O}_{2} \text { (control group) } 1 \mathrm{~h} \text { via } \\
\text { face mask }\end{array}$ & $\begin{array}{l}\text { Ar group had a significant reduction } \\
\text { in the overall, cortical, and basal } \\
\text { ganglia infarct volumes. Ar treatment } \\
\text { resulted in a significant improvement } \\
\text { of the composite adverse outcome }\end{array}$ & $\mathrm{N} / \mathrm{A}$ \\
\hline Zhao et al. (10) & $\mathrm{Ar}$ & Neonatal rats & $\begin{array}{l}\text { a) In vitro: Cortical } \\
\text { neuronal cell cultures } \\
\text { challenged by OGD } \\
\text { for } 90 \text { min } \\
\text { b) In vivo: } \\
\text { Rice-Vannucci } \\
\text { HI model }\end{array}$ & $\begin{array}{l}\text { a) Following OGD, cortical cells exposed to } \\
70 \% \text { Ar or } \mathrm{N}_{2} \text { with } 5 \% \mathrm{CO}_{2} \text { balanced } \\
\text { with } \mathrm{O}_{2} \text { at } 33^{\circ} \mathrm{C} \text { for } 2 \mathrm{~h} \\
\text { b) Following } \mathrm{HI} \text { : neonatal rats exposed to } \\
70 \% \text { Ar or } \mathrm{N}_{2} \text { balanced with } \mathrm{O}_{2} \text { at } 33 \text {, } \\
35 \text {, and } 37^{\circ} \mathrm{C} \text { for } 2 \mathrm{~h} \text {. Cortical and } \\
\text { hippocampal infarction size was } \\
\text { assessed at } 4 \text { weeks after treatment }\end{array}$ & $\begin{array}{l}\text { a) Ar-HT increased phospho-Akt and } \\
\text { HO1 expression and reduced the } \\
\text { Tyr216-GSK-3ß expression, } \\
\text { cytochrome C release, and cell } \\
\text { death in OGD-exposed cortical } \\
\text { neurons } \\
\text { b) Ar-HT treatment decreased infarct } \\
\text { size and activated both caspase-3 } \\
\text { and NF-kB in the cortex and } \\
\text { hippocampus } \\
\text { c) Ar-HT reduced hippocampal } \\
\text { astrocyte activation and } \\
\text { proliferation } \\
\text { d) Ar-HT inhibited the PI3K/Akt } \\
\text { pathway through LY294002 } \\
\text { attenuated cerebral protection }\end{array}$ & $\mathrm{N} / \mathrm{A}$ \\
\hline
\end{tabular}


TABLE 1 | Continued

\begin{tabular}{|c|c|c|c|c|c|c|}
\hline References & Gas & Age species & Procedure & Intervention & Outcomes & Behavioral \\
\hline $\begin{array}{l}\text { Zhuang et al. } \\
\text { (28) }\end{array}$ & $\begin{array}{l}\mathrm{Ar}, \mathrm{He} \\
\mathrm{Xe}\end{array}$ & Neonatal rats & Rice-Vannucci HI model & $\begin{array}{l}2 \mathrm{~h} \text { after } \mathrm{HI} \text { insult, animals were randomly } \\
\text { exposed } 90 \text { min to: } \\
\text { a) } \mathrm{Ar}, \mathrm{He}, \mathrm{Xe}(70 \% \text { noble gas balanced with } \\
\mathrm{O}_{2} \text { ), or } \\
\text { b) } \mathrm{N}_{2}\left(\text { control group, } 70 \% \mathrm{~N}_{2} \text { balanced }\right. \\
\text { with } \mathrm{O}_{2} \text { ) }\end{array}$ & $\begin{array}{l}\text { Ar improved cell survival to naiive } \\
\text { levels, whereas Xe and He did not. } \\
\text { When tested against more severe HI } \\
\text { injury only, Ar and Xe reduced infarct } \\
\text { volume. Ar, He, and Xe increased the } \\
\text { expression of Bcl-2, whereas He and } \\
\text { Xe increased Bcl-xL. In addition, Bax } \\
\text { expression was enhanced in the } \\
\text { control and He groups }\end{array}$ & N/A \\
\hline $\begin{array}{l}\text { Cheng et al. } \\
\text { (29) }\end{array}$ & $\mathrm{CO}$ & Neonatal mice & None & $\begin{array}{l}\text { For } 3 \mathrm{~h} \text { : } \\
\text { a) } 0 \text { ppm CO (air), } \\
\text { b) } 5 \text { ppm CO in air, or } \\
\text { c) } 100 \text { ppm CO in air }\end{array}$ & $\begin{array}{l}\text { a) } 3 \mathrm{~h} \text { exposure to } 5 \text { or } 100 \mathrm{ppm} \mathrm{CO} \\
\text { impaired cytochrome-c release, } \\
\text { caspase-3 activation, and } \\
\text { apoptosis in the neocortex and } \\
\text { hippocampus } \\
\text { b) CO increased NeuN protein and } \\
\text { neuronal numbers and resulted } \\
\text { in megalencephaly }\end{array}$ & $\begin{array}{l}\text { 4-5 weeks post exposure, mice } \\
\text { underwent Morris water maze, and } \\
\text { social approach-avoidance assay. } \\
\text { Escape latency was significantly } \\
\text { longer on day } 1 \text { and } 2 \text { of testing in } \\
\text { both CO-exposed cohorts compared } \\
\text { to controls. Air-exposed controls } \\
\text { showed a marked increase in } \\
\text { approach- avoidance score when the } \\
\text { stimulus mouse was placed in the } \\
\text { chamber }\end{array}$ \\
\hline $\begin{array}{l}\text { Queiroga et al. } \\
\text { (30) }\end{array}$ & $\mathrm{CO}$ & $\begin{array}{l}\text { Adult and } \\
\text { neonatal rats }\end{array}$ & $\begin{array}{l}\text { Apoptosis of astrocytes } \\
\text { induced by diamide }\end{array}$ & $\begin{array}{l}\text { Preconditioning with } \mathrm{CO} \text { prior to apoptosis } \\
\text { induction }\end{array}$ & $\begin{array}{l}\text { CO prevented membrane } \\
\text { depolarization induced by calcium } \\
\text { and inhibited mitochondrial swelling. } \\
\mathrm{CO} \text { prevented membrane pore } \\
\text { formation by increasing ANT activity }\end{array}$ & N/A \\
\hline $\begin{array}{l}\text { Queiroga et al. } \\
\text { (31) }\end{array}$ & $\mathrm{CO}$ & Neonatal rats & Rice-Vannucci HI model & $\begin{array}{l}\text { a) Sham surgery without hypoxia exposure } \\
\text { (Control group) } \\
\text { b) CO exposure prior to Sham surgery } \\
\text { without hypoxia exposure (CO+Sham } \\
\text { group) } \\
\text { c) } \mathrm{Hl} \text { group, } \\
\text { d) } \mathrm{CO} \text { exposure prior to } \mathrm{HI},(\mathrm{CO}+\mathrm{H} \text { group) }\end{array}$ & $\begin{array}{l}\text { a) } \mathrm{CO} \text { decreased apoptosis and } \\
\text { increased Bcl-2 mRNA in primary } \\
\text { cultures of neurons } \\
\text { b) CO decreased apoptosis in the } \\
\text { hippocampus, limited } \\
\text { cytochrome-c released from } \\
\text { mitochondria and reduced } \\
\text { activation of caspase-3 }\end{array}$ & N/A \\
\hline $\begin{array}{l}\text { Wang et al. } \\
\text { (32) }\end{array}$ & $\mathrm{CO}$ & $\begin{array}{l}\text { Adult male } \\
\text { mice }\end{array}$ & Permanent MCAO & $\begin{array}{l}\text { Administered for } 18 \mathrm{~h} \text { immediately after } \\
\text { permanent MCAO: } \\
\text { a) } 250 \text { ppm CO } \\
\text { b) control air }\end{array}$ & $\begin{array}{l}\text { a) Less brain damage than controls } \\
\text { at } 7 \text { days } \\
\text { b) } 18 \mathrm{~h} \text { CO treatment led to NRF2 } \\
\text { dissociation from Keap1, nuclear } \\
\text { translocation, increased binding } \\
\text { activity of NRF2 to HO1 ARE, and } \\
\text { elevated } \mathrm{HO} 1 \text { expression 6-48 } \mathrm{h} \\
\text { after } \mathrm{CO} \text { exposure } \\
\text { c) Loss of CO neuroprotection in } \\
\text { NRF2 knock-out mice }\end{array}$ & N/A \\
\hline
\end{tabular}


TABLE 1 | Continued

\begin{tabular}{|c|c|c|c|c|c|c|}
\hline References & Gas & Age species & Procedure & Intervention & Outcomes & Behavioral \\
\hline $\begin{array}{l}\text { Zeynalov and } \\
\text { Doré (33) }\end{array}$ & $\mathrm{CO}$ & Adult mice & $90 \mathrm{~min} \mathrm{MCAO}$ & $\begin{array}{l}\text { a) } 125 \mathrm{ppm} \text { normal air or } 250 \mathrm{ppm} \mathrm{CO} \text { at } \\
\text { onset of reperfusion } \\
\text { b) } 250 \mathrm{ppm} \text { CO inhalation } 1 \text { and } 3 \mathrm{~h} \\
\text { after reperfusion }\end{array}$ & $\begin{array}{l}\mathrm{CO} \text { inhalation reduced infarct size } \\
\text { and decreased brain edema }\end{array}$ & $\begin{array}{l}\text { Improved neurological deficit scores } \\
\text { at } 48 \mathrm{~h} \text { of survival time after ischemia }\end{array}$ \\
\hline Liu et al. (34) & $\mathrm{CO}$ & Neonatal rats & Rice-Vannucci HI model & $\begin{array}{l}\text { Eight groups: } \\
\text { a) Sham } \\
\text { b) Sham + electroacupuncture } \\
\text { c) } \mathrm{HIE} \\
\text { d) } \mathrm{HIE}+\text { electroacupuncture } \\
\text { e) } \mathrm{HIE}+\mathrm{SAM} \\
\text { f) } \mathrm{HIBD}+\mathrm{SAM}+\text { electroacupuncture } \\
\text { g) } \mathrm{HIE}+\mathrm{HA} \\
\text { h) } \mathrm{HIE}+\mathrm{HA}+\text { electroacupuncture }\end{array}$ & $\begin{array}{l}\text { a) Electroacupuncture significantly } \\
\text { downregulated the expression of } \\
\text { nNOS and NF-kB in the rat cortex } \\
\text { cells and alleviated cortical atrophy } \\
\text { caused by HIE } \\
\text { b) Increased intrinsic CO levels via } \\
\text { overexpression of } \\
\text { hemoxygenase-1 (HO1) in the } \\
\text { cortex and was associated with } \\
\text { alleviated cortical damage }\end{array}$ & N/A \\
\hline $\begin{array}{l}\text { Kohzuki et al. } \\
\text { (35) }\end{array}$ & $\mathrm{CO}_{2}$ & Neonatal rats & Rice-Vannucci HI model & $6 \% \mathrm{CO}_{2}$ delivered during $\mathrm{HI}$ & $\begin{array}{l}\text { Smaller brain infarct size in rats } \\
\text { exposed to } \mathrm{CO}_{2}\end{array}$ & $\begin{array}{l}\text { Staircase test at } 3 \text { months of age } \\
\text { showed improved forelimb strength in } \\
\text { rats exposed to } \mathrm{CO}_{2}\end{array}$ \\
\hline $\begin{array}{l}\text { Vannucc et al. } \\
\text { (36) }\end{array}$ & $\mathrm{CO}_{2}$ & Neonatal rats & Rice-Vannucci HI model & $\begin{array}{l}\text { a) } 0 \% \text { (hypocapnia) } \\
\text { b) } 3 \% \text { (normocapnia) } \\
\text { c) } 6 \% \mathrm{CO}_{2} \text { (hypercapnia), } \\
\text { all with } 8 \% \mathrm{O}_{2} \text {, balanced with } \mathrm{N}_{2}\end{array}$ & $\begin{array}{l}\text { a) Hypocapnia was associated with } \\
\text { decreased cerebral blood flow } \\
\text { b) Normocapnia and hypercapnia } \\
\text { groups showed preservation of } \\
\text { cerebral blood flow in addition to } \\
\text { maintaining higher cerebral } \\
\text { glucose and lower } \\
\text { lactate concentrations }\end{array}$ & N/A \\
\hline $\begin{array}{l}\text { Vannucc et al. } \\
\text { (37) }\end{array}$ & $\mathrm{CO}_{2}$ & Neonatal rats & Rice-Vannucci HI model & 3,12 , or $15 \% \mathrm{CO}_{2}$ for $2 \mathrm{~h}$ at $37^{\circ} \mathrm{C}$ & $\begin{array}{l}\text { a) Inhaled } 12 \text { and } 15 \% \mathrm{CO}_{2} \text { was } \\
\text { associated with blood } \mathrm{CO}_{2} \\
\text { tension of } 80 \text { and } 100 \mathrm{mmHg} \text {, } \\
\text { respectively } \\
\text { b) Extreme hypercapnia }\left(15 \% \mathrm{CO}_{2}\right) \\
\text { was associated with severe brain } \\
\text { infarcts following } \mathrm{HI} \text { insult and } \\
\text { significant reduction of cerebral } \\
\text { blood flow when compared to the } \\
\text { other groups }\end{array}$ & N/A \\
\hline $\begin{array}{l}\text { Vannucc et al. } \\
\text { (38) }\end{array}$ & $\mathrm{CO}_{2}$ & Neonatal rats & Rice-Vannucci HI model & $0,3,6$, or $9 \% \mathrm{CO}_{2}$ & $\begin{array}{l}\text { a) Mild hypercapnia }\left(6 \% \mathrm{CO}_{2}\right) \text { had } \\
\text { the lowest brain atrophy followed } \\
\text { by normocapnia }\left(3 \% \mathrm{CO}_{2}\right) \text {, while } \\
\text { hypocapnia }\left(0 \% \mathrm{CO}_{2}\right) \text { had the } \\
\text { worst damage } \\
\text { b) } \mathrm{CO}_{2} \text { exposure was associated } \\
\text { with lower lactate levels and } \\
\text { improved glucose concentrations } \\
\text { by preventing hypoglycemia }\end{array}$ & $\mathrm{N} / \mathrm{A}$ \\
\hline
\end{tabular}


TABLE 1 | Continued

\begin{tabular}{|c|c|c|c|c|c|c|}
\hline References & Gas & Age species & Procedure & Intervention & Outcomes & Behavioral \\
\hline Cai et al. (39) & $\mathrm{H}_{2}$ & Neonatal rats & Rice-Vannucci HI model & $\begin{array}{l}\text { Neonatal rats: Intra-ischemic treatment and } \\
\text { post-ischemic treatment } \\
\text { a) Sham } \\
\text { b) } \mathrm{HI} \\
\text { c) } \mathrm{HI}+\mathrm{H}_{2} \\
\text { Adult rats: } \\
\text { a) MCAO } \\
\text { b) MCAO+ } \mathrm{H}_{2}\end{array}$ & $\begin{array}{l}\mathrm{H}_{2} \text { treatment significantly reduced } \\
\text { the number of positive TUNEL cells } \\
\text { and suppressed caspase- } 3 \text { and }-12 \\
\text { activities }\end{array}$ & N/A \\
\hline $\begin{array}{l}\text { Matchett et al. } \\
(40)\end{array}$ & $\mathrm{H}_{2}$ & $\begin{array}{l}\text { Neonatal and } \\
\text { adult rats }\end{array}$ & $\begin{array}{l}\text { Neonatal rats: } \\
\text { Modified Rice-Vannucci } \\
\text { HI model } 120 \text { and } \\
150 \text { min } \\
\text { Adult rats: MCAO }\end{array}$ & $\begin{array}{l}\text { Neonatal } \mathrm{Hl} \text { : } \\
\text { a) Sham } \\
\text { b) Sham }+ \text { intra-ischemia } \mathrm{H}_{2} \times 2 \mathrm{~h} \\
\text { c) } \mathrm{HI} \text { only } \\
\text { d) } \mathrm{H}+\text { Preconditioning with } \mathrm{H}_{2} \times 1.5 \mathrm{~h} \\
\text { e) } \mathrm{HI}+\text { post-ischemia } \mathrm{H}_{2} \times 1 \mathrm{~h} \\
\text { Adult MCAO: } \\
\text { a) Sham } \\
\text { b) } \mathrm{HI} \text { only } \\
\text { c) } \mathrm{HI}+\text { intra-ischemia } \mathrm{H}_{2} \times 2.5 \mathrm{~h} \\
\text { d) } \mathrm{HI} \text { post-ischemia } \mathrm{H}_{2} \times 1 \mathrm{~h} \\
\text { e) } \mathrm{HI}+\text { post-operative-ischemia } \times 1.5 \mathrm{~h}\end{array}$ & $\begin{array}{l}\text { a) } \mathrm{H}_{2} \text { therapy in neonatal } \mathrm{HI} \text { was not } \\
\text { associated with decreased volume } \\
\text { of infarction or decreased } \\
\text { concentration of MDA } \\
\text { b) } \mathrm{H}_{2} \text { pretreatment was associated } \\
\text { with increased infarction volume in } \\
\text { neonatal } \mathrm{HI} \\
\text { c) Exposure of } \mathrm{H}_{2} \text { to non-ischemic } \\
\text { neonates was associated with a } \\
\text { significant increase in brain } \\
\text { concentration of MDA }\end{array}$ & N/A \\
\hline $\begin{array}{l}\text { Ohsawa et al. } \\
\text { (41) }\end{array}$ & $\mathrm{H}_{2}$ & Adult rats & $\begin{array}{l}\text { In vitro: neuronal cell } \\
\text { culture treated with } \\
\text { a) antimycin A and } \\
\text { menadione } \\
\text { b) OGD In vivo: Adult } \\
\text { male rats underwent } \\
\text { MCAO } \times 90 \text { min } \\
\text { followed by } \\
\text { reperfusion } \times 30 \text { min }\end{array}$ & $\begin{array}{l}\text { Four groups: } \\
\text { a) } 1 \% \mathrm{H}_{2} / 30 \% \mathrm{O}_{2} / 69 \% \mathrm{~N}_{2} \mathrm{O} \\
\text { b) } 2 \% \mathrm{H}_{2} / 30 \% \mathrm{O}_{2} / 68 \% \mathrm{~N}_{2} \mathrm{O} \\
\text { c) } 4 \% \mathrm{H}_{2} / 30 \% \mathrm{O}_{2} / 66 \% \mathrm{~N}_{2} \mathrm{O} \\
0 \% \mathrm{H}_{2} / 30 \% \mathrm{O}_{2} / 70 \% \mathrm{~N}_{2} \mathrm{O} \mathrm{H}_{2} \text { was } \\
\text { administered either for the entire } \\
\text { procedure (120 min), last } 35 \text { min, or } \\
\text { first } 85 \text { min }\end{array}$ & $\begin{array}{l}\text { a) Decreased oxidative stress and } \\
\text { infarction } \\
\text { b) Reduction of cytotoxic radicals } \\
\text { c) Induced cytoprotective factors } \\
\text { that prolong cell life }\end{array}$ & N/A \\
\hline Li et al. (42) & $\mathrm{He}$ & Neonatal rats & Rice-Vannucci HI model & $\begin{array}{l}\text { a) Normal control group } \\
\text { b) He-Preconditioning group }(70 \% \mathrm{He} / 30 \% \\
\mathrm{O}_{2} \text { for three } 5 \text {-min periods) } \\
\text { c) } \mathrm{HIE} \text { group } \\
\text { d) He-Preconditioning + HIE group } \\
\text { e) L-NAME + HIE group } \\
\text { f) L- NAME + He-Preconditioning + } \\
\text { HIE group }\end{array}$ & $\begin{array}{l}\text { a) Reduction in infarct size and } \\
\text { increase in NO content in the brain } \\
\text { b) Increased anti-oxidase expression } \\
\text { and DNA binding activity of } \\
\text { NRF-2. Suggests that } \\
\text { preconditioning promotes intrinsic } \\
\text { NO production that } \\
\text { is neuroprotective }\end{array}$ & $\begin{array}{l}\text { Tested at } 3 \text { weeks. } \\
\text { He-preconditioning group had better } \\
\text { scores in the wire hang and beam } \\
\text { balance tests compared to the HIE } \\
\text { group. }\end{array}$ \\
\hline
\end{tabular}


TABLE 1 | Continued

\begin{tabular}{|c|c|c|c|c|c|c|}
\hline References & Gas & Age species & Procedure & Intervention & Outcomes & Behavioral \\
\hline Pan et al. (43) & $\mathrm{He}$ & $\begin{array}{l}\text { Adult male } \\
\text { rats }\end{array}$ & $\begin{array}{l}\text { MCAO for } 2 \mathrm{~h} \text { and } \mathrm{a} 1 \mathrm{~h} \\
\text { reperfusion }\end{array}$ & $\begin{array}{l}\text { During stroke and reperfusion rats were } \\
\text { subjected to either: } \\
\text { a) } 30 \% \mathrm{O}_{2} / 70 \% \mathrm{~N}_{2} \text { (control group) } \\
\text { b) } 100 \% \mathrm{O}_{2} \text { (hyperoxia group), OR } \\
\text { c) } 30 \% \mathrm{O}_{2} / 70 \% \mathrm{He} \text { (heliox group) }\end{array}$ & $\begin{array}{l}\text { a) Infarct volume in the heliox group } \\
\text { was smaller than the hyperoxia } \\
\text { and control groups } \\
\text { b) Neurologic scores in the heliox } \\
\text { group were significantly better } \\
\text { compared with controls }\end{array}$ & $\begin{array}{l}\text { Neurologic scores } 1 \text { and } 24 \mathrm{~h} \text { post } \\
\text { MCAO in the heliox group were } \\
\text { significantly better compared with } \\
\text { controls using Hunter neurological } \\
\text { scores }\end{array}$ \\
\hline Li et al. (44) & NO & Adult mice & $\begin{array}{l}\text { Transient MCAO for } 1 \mathrm{~h} \\
\text { followed by reperfusion } \\
\text { for } 47 \mathrm{~h}\end{array}$ & $\begin{array}{l}\text { Inhaled } \mathrm{NO} \text { at } 10,20,40,60 \text {, or } 80 \mathrm{ppm} \text {. } \\
\text { Each group was subdivided into four } \\
\text { duration groups for } 5,8,16 \text {, or } 24 \mathrm{~h} \\
\text { beginning immediately after MCAO }\end{array}$ & $\begin{array}{l}\text { a) At } 10 \mathrm{ppm} \text { only the } 24 \mathrm{~h} \text { duration } \\
\text { group exhibited reduced infarct } \\
\text { volume } \\
\text { b) At } 20,40 \text {, and } 60 \mathrm{ppm} \text {, only } 8 \text { and } \\
16 \mathrm{~h} \text { of exposure led to smaller } \\
\text { infarctions } \\
\text { c) } 60 \mathrm{ppm} \text { iNO could be transported } \\
\text { from lung to brain } \\
\text { d) iNO administered for } 8 \mathrm{~h} \text { improved } \\
\text { recovery from subarachnoid } \\
\text { hemorrhage and reduced the } \\
\text { inflammatory response } \\
\text { accompanying ischemic stroke }\end{array}$ & N/A \\
\hline Lu et al. (45) & NO & Neonatal rats & $\begin{array}{l}\text { a) Neuronal cells } \\
\text { underwent OGD } \times \\
90 \text { min } \\
\text { b) Modified } \\
\text { Rice-Vannucci } \\
\text { HI model }\end{array}$ & No intervention & $\begin{array}{l}\text { a) OGD increased NO generation } \\
\text { b) HA disrupted iron regulation } \\
\text { c) Hydroxyl radicals and iron } \\
\text { deposition were ameliorated by } \\
\text { NOS- inhibited aconitase activity } \\
\text { and lead to cellular } \\
\text { iron accumulation }\end{array}$ & N/A \\
\hline $\begin{array}{l}\text { Terpolilli et al. } \\
\text { (46) }\end{array}$ & NO & $\begin{array}{l}\text { Adult male } \\
\text { rats, Adult } \\
\text { male mice, } \\
\text { and Neonatal } \\
\text { mice }\end{array}$ & $\begin{array}{l}\text { a) Adult rats underwent } \\
\text { MCAO } \\
\text { b) Adult mice } \\
\text { underwent bilateral } \\
\text { carotid banding } \\
\text { c) Neonatal mice } \\
\text { ligature of the left } \\
\text { common carotid } \\
\text { artery, } 1 \mathrm{~h} \text { later, } \\
\text { hypoxia for } 50 \text { min } \\
\text { with or without } \\
\text { addition of } 50 \text { ppm } \\
\text { NO to the gas mixture }\end{array}$ & 50 ppm NO & $\begin{array}{l}\text { a) In adult mice, inhaled NO } \\
\text { enhanced blood flow during } \\
\text { reperfusion and reduced } \\
\text { inflammation } \\
\text { b) Pups that received iNO at the time } \\
\text { of hypoxic injury had smaller } \\
\text { infarct volume and lower } \\
\text { histopathological scores } \\
\text { compared to controls }\end{array}$ & N/A \\
\hline Zhu et al. (47) & NO & Neonatal mice & Rice-Vannucci HI model & $\begin{array}{l}50 \mathrm{ppm} N \mathrm{NO} \text { mixed with } \mathrm{N}_{2} \text { vs. } \mathrm{N}_{2} \text { alone for } \\
50 \mathrm{~min}\end{array}$ & $\begin{array}{l}\text { Reduced brain injury and tissue loss. } \\
\text { Only male mice had significant } \\
\text { reduction of infarct size }\end{array}$ & N/A \\
\hline $\begin{array}{l}\text { Calvert et al. } \\
(48)\end{array}$ & $\mathrm{O}_{2}$ & Neonatal rats & Rice-Vannucci HI model & $\begin{array}{l}\text { Three groups: } \\
\text { a) } \mathrm{HI} \\
\text { b) } \mathrm{HI} \text { and } \mathrm{HBO} ; 100 \% \mathrm{O}_{2} \text { at } 3 \text { ATA for } 1 \mathrm{~h} \\
\text { (following recovery for } 1 \mathrm{~h} \text { ) } \\
\text { c) Control (no anesthesia, carotid ligation, } \\
\text { hypoxia, or HBO exposure) }\end{array}$ & $\begin{array}{l}\text { HBO decreased caspase- } 3 \text { activity, } \\
\text { PARP cleavage and DNA } \\
\text { fragmentation. HBO preserved brain } \\
\text { weight. }\end{array}$ & N/A \\
\hline
\end{tabular}


TABLE 1 | Continued

\begin{tabular}{|c|c|c|c|c|c|c|}
\hline References & Gas & Age species & Procedure & Intervention & Outcomes & Behavioral \\
\hline $\begin{array}{l}\text { Dalen et al. } \\
\text { (49) }\end{array}$ & $\mathrm{O}_{2}$ & Neonatal rats & $\begin{array}{l}\text { Rice-Vannucci HA } \\
\text { model }\end{array}$ & $\begin{array}{l}\mathrm{Hl} \text { followed by } 30 \text { min reoxygenation in } 21 \% \\
\mathrm{O}_{2} \text { or } 100 \% \mathrm{O}_{2} \text { before } 5 \mathrm{~h} \text { of } \mathrm{NT}\left(37^{\circ} \mathrm{C}\right) \text { or } \\
\mathrm{HT}\left(32^{\circ} \mathrm{C}\right)\end{array}$ & $\begin{array}{l}\text { Reoxygenation with } 100 \% \mathrm{O}_{2} \\
\text { increased hippocampal injury score } \\
\text { and negated } \mathrm{HT} \text { neuroprotection }\end{array}$ & $\begin{array}{l}\text { Reoxygenation with } 100 \% \mathrm{O}_{2} \\
\text { worsened reflex performance in } \\
\text { staircase test }\end{array}$ \\
\hline Smit et al. (50) & $\mathrm{O}_{2}$ & Neonatal rats & $\begin{array}{l}\text { Modified Rice-Vannucci } \\
\text { HI model }\end{array}$ & $\begin{array}{l}\text { Soon after } \mathrm{HI} \text {, pups underwent immediate } \\
\text { resuscitation in either } 21 \text { or } 100 \% \mathrm{O}_{2} \text { for } \\
30 \text { min }\end{array}$ & No significant change in brain atrophy & $\begin{array}{l}\text { No change in short-term neurologic } \\
\text { outcome }\end{array}$ \\
\hline $\begin{array}{l}\text { Dingley et al. } \\
\text { (51) }\end{array}$ & $\mathrm{Xe}$ & Neonatal rats & Rice-Vannucci HI model & $\begin{array}{l}\text { After } \mathrm{Hl} \text { insult, } 3 \mathrm{~h} \text { inhalation of } \\
\text { a) } 50 \% \mathrm{Xe}_{\mathrm{e}} / 30 \% \mathrm{O}_{2} / 20 \% \mathrm{~N}_{2} \\
\text { b) } 30 \% \mathrm{O}_{2} / 70 \% \mathrm{~N}_{2}\end{array}$ & $\begin{array}{l}\text { a) One week after HI survival, } \\
\text { significant global protection in the } \\
\text { Xe group ( } 80 \% \text { less injury) } \\
\text { b) Percentage of global damage } \\
\text { score in non-Xe vs. Xe groups: } \\
\text { cortex/white matter ( } 88 \text { vs. } 25 \% \text { ); } \\
\text { hippocampus ( } 62 \text { vs. } 0 \% \text { ); basal } \\
\text { ganglia ( } 81 \text { vs. } 25 \% \text { ); and } \\
\text { thalamus ( } 50 \text { vs. } 0 \% \text { ), respectively }\end{array}$ & N/A \\
\hline $\begin{array}{l}\text { Hobbs et al. } \\
\text { (12) }\end{array}$ & $\mathrm{Xe}$ & Neonatal rats & Rice-Vannucci HI model & $\begin{array}{l}\text { a) } \mathrm{NT} 37^{\circ} \mathrm{C} \\
\text { b) } H T 32^{\circ} \mathrm{C} \\
\text { c) } \mathrm{Xe} 50 \%+\mathrm{NT} 37^{\circ} \mathrm{C} \\
\text { d) } \mathrm{Xe} 50 \%+H T 32^{\circ} \mathrm{C}\end{array}$ & $\begin{array}{l}\text { a) } \mathrm{Xe} 50 \%+\mathrm{HT} 32^{\circ} \mathrm{C} \text { produced the } \\
\text { greatest improvement }(71 \%) \text { in } \\
\text { global histopathology scores. The } \\
\text { overall effect of } \mathrm{HT} \text { and } \mathrm{Xe} \text { is } \\
\text { additive) } \mathrm{Xe} 50 \% \text { and } \mathrm{HT} 32^{\circ} \mathrm{C} \\
\text { individually produced smaller } \\
\text { improvements }\end{array}$ & $\begin{array}{l}\text { Staircase testing (long-term) was } \\
\text { performed from } 8 \text { to } 11 \text { weeks. } \\
\text { Results: } \\
\text { a) } \mathrm{Xe} 50 \%+\mathrm{HT} 32^{\circ} \mathrm{C} \text { group: } \\
\text { complete restoration of long-term } \\
\text { functional outcomes } \\
\text { b) Untreated pups }\left(\mathrm{NT} 37^{\circ} \mathrm{C} \text { ) reached }\right. \\
\text { an early performance plateau of } \\
\text { staircase test } \\
\text { c) The Xe } 50 \%+\mathrm{NT} 37^{\circ} \mathrm{C} \text { or } \mathrm{HT} 32^{\circ} \mathrm{C} \\
\text { only groups, had mild } \\
\text { improvement in staircase testing }\end{array}$ \\
\hline Luo et al. (52) & $\mathrm{xe}$ & Neonatal mice & $\begin{array}{l}\text { a) In vitro: OGD } 1 \mathrm{~h} \text { after } \\
\text { preconditioning of } \\
\text { cells for } 2 \mathrm{~h} \\
\text { b) In vivo: } 4 \mathrm{~h} \text { after } \\
\text { preconditioning for } \\
120 \text { min, mice } \\
\text { underwent } \\
\text { Rice-Vannucci } \\
\text { HI model }\end{array}$ & $\begin{array}{l}\text { In vitro: Neuronal cells were preconditioned } \\
\text { for } 120 \text { min with } 12.5,25,50 \text {, or } 75 \% \text { Xe, } \\
0.67-3.3 \% \text { sevoflurane, or a combination of } \\
\text { both } \\
\text { In vivo: Neonatal mice were preconditioned } \\
\text { with } 20 \text { or } 75 \% \text { Xe, } 0.75 \text { or } 1.5 \% \\
\text { sevoflurane, or } 20 \% \text { Xe plus } 0.75 \% \\
\text { sevoflurane in } 25 \% \mathrm{O}_{2} \text { balanced with } \mathrm{N}_{2}\end{array}$ & $\begin{array}{l}\text { a) In vitro: Preconditioning with Xe for } \\
2 \mathrm{~h} \text { produces a } \\
\text { concentration-dependent } \\
\text { reduction in } \mathrm{LDH} \text { release after } \\
\text { OGD. } \mathrm{LDH} \text { release was } \\
\text { significantly reduced by } \mathrm{Xe} \\
\text { concentrations of } 50 \text { and } 75 \% \\
\text { b) In vivo: Although sevoflurane alone } \\
\text { did not reduce infarct size in } \\
\text { Rice-Vannucci model of neonatal } \\
\mathrm{HI} \text { injury, Xe alone or in } \\
\text { combination with sevoflurane } \\
\text { reduced infarct size and improved } \\
\text { neuromotor function at } 30 \text { days } \\
\text { with slight advantage of the } \\
\text { second group }\end{array}$ & N/A \\
\hline
\end{tabular}


TABLE 1 | Continued

\begin{tabular}{|c|c|c|c|c|c|c|}
\hline References & Gas & Age species & Procedure & Intervention & Outcomes & Behavioral \\
\hline Ma et al. (53) & $x_{e}$ & Adult mice & $\begin{array}{l}24 \mathrm{~h} \\
\text { after preconditioning: } \\
\text { a) bilateral renal pedicle } \\
\text { clamping for } 25 \text { min } \\
\text { or } \\
\text { b) right renal pedicle } \\
\text { clamping for } 40 \text { min } \\
\text { and left nephrectomy } \\
\text { c) Sham-operated mice } \\
\text { had dissection as } \\
\text { above but with no } \\
\text { occlusion of the } \\
\text { renal vessel }\end{array}$ & $\begin{array}{l}\text { Preconditioning for } 3 \mathrm{~h} \text { with } 70 \% \mathrm{Xe}, 70 \% \\
\mathrm{~N}_{2} \text {, or } 70 \% \mathrm{~N}_{2} \mathrm{O} \text { balanced with } 30 \% \mathrm{O}_{2} \text { for } \\
2 \mathrm{~h} \text { or } 8 \% \mathrm{O}_{2} \text { balanced with } \mathrm{N}_{2}\end{array}$ & $\begin{array}{l}\text { Xe preconditioning protects against } \\
\text { intermittent renal ischemia through } \\
\text { increased expression of } \mathrm{HIF-1 \alpha} \\
\text { Knockout mice lost protective effect }\end{array}$ & $\mathrm{N} / \mathrm{A}$ \\
\hline Ma et al. (54) & $\mathrm{xe}$ & Neonatal rats & $\begin{array}{l}\text { In vitro: cell culture OGD } \\
\text { In vivo: Rice-Vannucci } \\
\text { HA model }\end{array}$ & $\begin{array}{l}\text { In vitro: Neuronal culture cells were } \\
\text { preconditioned with Xe for } 2 \mathrm{~h} \text {. } \\
\text { In vivo: } \\
\text { a) Treatment group was preconditioned with } \\
70 \% \mathrm{Xe}+30 \% \mathrm{O}_{2} \text { for } 120 \text { min } \\
\text { b) Control group was exposed to } 70 \% \mathrm{~N}_{2} \mathrm{O} \\
\text { balanced with } \mathrm{O}_{2} \text { for } 120 \text { min }\end{array}$ & $\begin{array}{l}\text { a) Concentration-dependent } \\
\text { reduction of } L D H \text { release from } \\
\text { cells with OGD } 24 \mathrm{~h} \text { later } \\
\text { b) Preconditioning with Xe } \\
\text { decreased propidium iodide } \\
\text { staining in a hippocampal slice } \\
\text { culture model subjected to OGD } \\
\text { c) Preconditioning with Xe reduced } \\
\text { infarction size when assessed } 7 \\
\text { days after injury } \\
\text { d) pCREB was increased by } \\
\text { Xe exposure }\end{array}$ & $\mathrm{N} / \mathrm{A}$ \\
\hline $\begin{array}{l}\text { Martin et al. } \\
\text { (13) }\end{array}$ & $x e$ & Neonatal rats & Rice-Vannucci HI model & $\begin{array}{l}\text { After a } 1 \mathrm{~h} \text { recovery period, rats received } \\
\text { asynchronous administration of } \\
\text { a) Mild } \mathrm{HT}\left(35.8^{\circ} \mathrm{C}\right) \text { and } 20 \% \text { Xe with a } 1 \text { - or } \\
5 \text {-h gap between interventions } \\
\text { b) } 20 \% \mathrm{Xe} \text { alone } \\
\text { c) Mild } \mathrm{HT}\left(35.8^{\circ} \mathrm{C}\right) \text { alone }\end{array}$ & $\begin{array}{l}\text { a) Brain infarct was reduced by } \mathrm{Xe} \\
\text { and } \mathrm{HT} \text { administration at } 1 \mathrm{~h} \\
\text { intervals after } 90 \text { min of } \mathrm{HI} \\
\text { b) Administration of } 20 \% \text { Xe after } 6 \mathrm{~h} \\
\text { of } \mathrm{HI} \text { and } 2 \text { - } \mathrm{h} \text { administration of } \\
35.8^{\circ} \mathrm{C} \mathrm{HT} 1 \mathrm{~h} \text { following the } \mathrm{HI} \\
\text { insult was not neuroprotective }\end{array}$ & N/A \\
\hline Sabir et al. (14) & $\mathrm{xe}$ & Neonatal rats & Rice-Vannucci HI model & $\begin{array}{l}\text { a) Immediate } \mathrm{NT}-37^{\circ} \mathrm{C} \text { for } 5 \mathrm{~h} \\
\text { b) Immediate } \mathrm{HT}-32^{\circ} \mathrm{C} \text { : Trectal } 32^{\circ} \mathrm{C} \text { for } 5 \mathrm{~h} \\
\text { c) Immediate } \mathrm{HT}-32^{\circ} \mathrm{C} \text { plus } 50 \% \text { inhaled } \mathrm{Xe} \\
\text { for } 5 \mathrm{~h}\end{array}$ & $\begin{array}{l}\text { a) No difference in neuronal cell } \\
\text { count in the subventricular zone } \\
\text { among different treatment groups } \\
\text { b) No reduction in brain area loss in } \\
\text { either the HT group or in the HT } \\
\text { plus } 50 \% \text { inhaled Xe group }\end{array}$ & N/A \\
\hline Sabir et al. (55) & $\mathrm{xe}$ & Neonatal rats & Rice-Vannucci HI model & 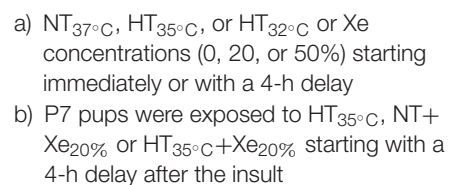 & $\begin{array}{l}\text { a) Immediate } \mathrm{HT} \text { to } 32^{\circ} \mathrm{C} \text { but not } \\
35^{\circ} \mathrm{C} \text { significantly reduced infarct } \\
\text { size } \\
\text { b) Synergistic effect when } \mathrm{Xe} \\
\text { immediately added to } \mathrm{HT}_{32^{\circ} \mathrm{C}}\end{array}$ & $\mathrm{N} / \mathrm{A}$ \\
\hline
\end{tabular}




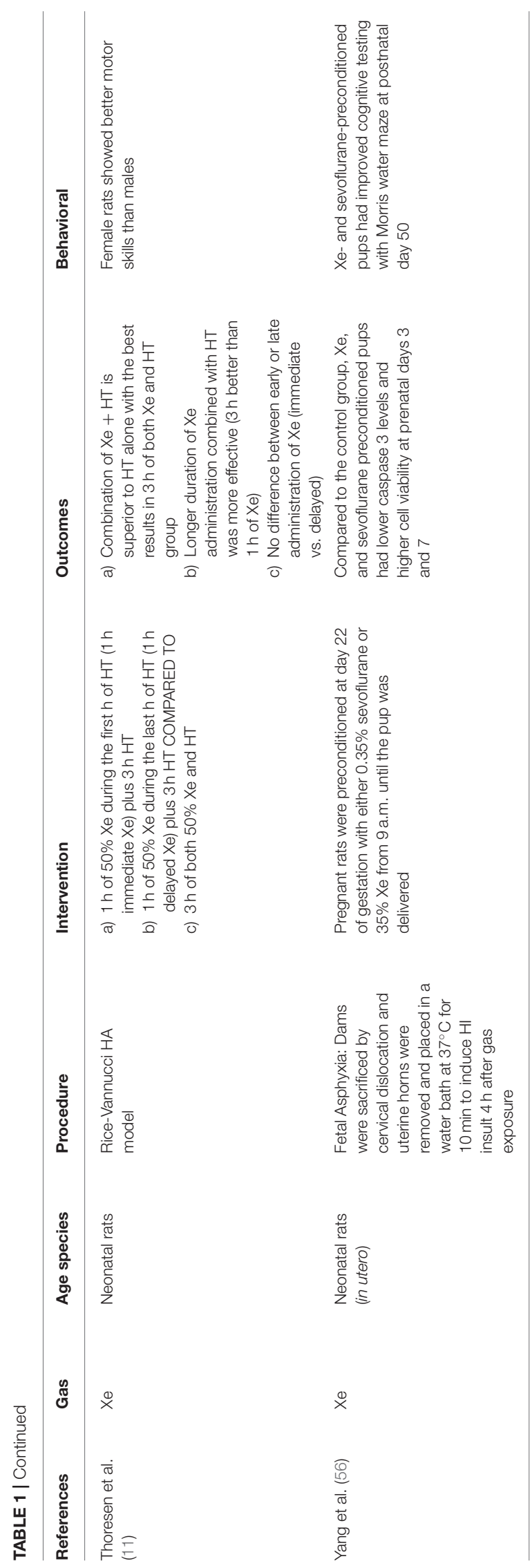

\section{Clinical Studies}

A feasibility study combined xenon with hypothermia in a single-arm, dose-escalation design. The dose of Xenon was increased from 25 to $50 \%$. The study did not find adverse respiratory or cardiovascular effects when xenon was combined with hypothermia (51). Administering 50\% Xenon would limit fractional inspired oxygen delivery to infants to $50 \%$ which may restrict its clinical use in neonates with HIE that have hypoxemic respiratory failure or persistent pulmonary hypertension.

"Total Body hypothermia plus Xenon (TOBY-Xe)" was a randomized, open-label, parallel-group trial done in 4 Neonatal intensive care units in the UK (NCT 00934700). Within $12 \mathrm{~h}$ of birth, patients with moderate to severe HIE were randomized to either hypothermia $\left(33.5^{\circ} \mathrm{C}\right)$ for $72 \mathrm{~h}$ or cooling combined with $30 \%$ inhaled xenon for $24 \mathrm{~h}$. Two primary outcomes were measured within 15 days of birth. First, the lactate/NAA ratio in the thalamus was measured with magnetic resonance spectroscopy and was reduced. Unlike lactate which rises as a result of ischemia, $\mathrm{N}$-acetyl aspartate (NAA) is an amino acid found naturally in the brain. Elevation of lactate/NAA is a marker of brain hypoxic-ischemic injury. Second, the fractional anisotropy in the posterior limb of the internal capsule was measured with MRI and was preserved. Similarly to the lactate/NAA ratio, fractional anisotropy is a marker of injury to the white matter tracts after hypoxic-ischemic injury. The study did not show statistical differences between the two groups in the lactate to NAA ratio in the thalamus or in the fractional anisotropy in the posterior limb of the internal capsule (15). Lack of effect of xenon may be related to the delayed administration of xenon to neonates with HIE at a median of $10 \mathrm{~h}$ of age. Additionally, the authors also reported that $30 \%$ xenon which is sub-anesthetic dosing (anesthetic dosing is achieved with alveolar xenon concentration of $60 \%$ ) was effective in suppressing seizures associated with HIE, which is an effect that was not previously reported (78).

"Xenon and Cooling Therapy in Babies at High Risk of Brain Injury Following Poor Condition at Birth (CoolXenon3)" is an ongoing clinical trial evaluating the benefit of inhaled xenon gas treating newborn infants with HIE in combination with cooling (NCT02071394). The study is comparing $72 \mathrm{~h}$ of whole-body hypothermia starting within $3 \mathrm{~h}$ after birth vs. wholebody hypothermia in addition to $50 \%$ xenon inhalation via endotracheal tube for $18 \mathrm{~h}$ starting within $5 \mathrm{~h}$ after birth. The primary outcome of the study is death or moderate to severe disability at 18 months of age.

\section{Argon}

The interest in studying other noble gases such as argon grew after xenon emerged as a neuroprotective agent. Unlike xenon, argon is more abundant and cheaper to manufacture, and it is not a sedative. Argon's mechanism of action for neuroprotection is not well-understood. The blockage of GABA receptors at high atmospheric pressure is a proposed mechanism of neuroprotection (79). Argon has no effect on heart rate, blood pressure, cerebral oxygen saturation, or blood gas in a preclinical piglet study (57). 
TABLE 2 | Summary of preclinical studies of inhaled gases in large animal models.

\begin{tabular}{|c|c|c|c|c|c|c|}
\hline References & Gas & Age species & Procedure & Intervention & Outcomes & Behavioral \\
\hline Alderliesten et al. (57) & $\operatorname{Ar}$ & Newborn piglets & Hypoxia by inhaling $8 \% \mathrm{O}_{2} \times 1 \mathrm{~h}$ & $\begin{array}{l}\text { a) Titration up from } 30 \% \mathrm{Ar} \times 1 \mathrm{~h} \\
\text { then } 50 \% \mathrm{Ar} \times 1 \mathrm{~h} \text { to } 80 \% \mathrm{Ar} \\
\times 1 \mathrm{~h} \\
\text { b) Hypoxia }+50 \% \mathrm{Ar} \times 3 \text { periods } \\
\text { of } 1 \mathrm{~h} \\
\text { c) Hypoxia+hypothermia }+50 \% \\
\mathrm{Ar} \times 3 \text { periods of } 1 \mathrm{~h} \\
\text { d) Cage animal control }\end{array}$ & $\begin{array}{l}\text { No hemodynamic instability in Ar group (HR, } \\
\text { BP, cerebral oxygen saturation, or blood gas) }\end{array}$ & $N / A$ \\
\hline Broad et al. (58) & $\mathrm{Ar}$ & Newborn piglets & $\begin{array}{l}\text { Occluding common carotid } \\
\text { arteries bilaterally and reducing } \\
\text { inspired } \mathrm{FiO}_{2} \text { to } 6 \%\end{array}$ & $\begin{array}{l}\text { a) Hypothermia }(\mathrm{HT}) \text { to } 33.5^{\circ} \mathrm{C} \\
\times 24 \mathrm{~h} \\
\text { b) } \mathrm{HT}+45-50 \% \mathrm{Ar} \times 24 \mathrm{~h}\end{array}$ & $\begin{array}{l}\text { HT with argon preserved brain MRS (magnetic } \\
\text { resonance spectroscopy) ATP, decreased MRS } \\
\text { lactate/NAA peak, and expedited EEG } \\
\text { background recovery when compared to HT } \\
\text { alone }\end{array}$ & N/A \\
\hline Oláh et al. (59) & $\mathrm{H}_{2}$ & Newborn piglets & $\begin{array}{l}\text { Asphyxia by clamping ET tube } \\
x 8 \text { min }\end{array}$ & $\begin{array}{l}\text { a) Time control } \\
\text { b) Asphyxia+RA (room air) } \\
\text { c) Asphyxia+2.1\% } \mathrm{H}_{2} \times 4 \mathrm{~h}\end{array}$ & $\begin{array}{l}\text { a) } \mathrm{H}_{2} \text {-treated animals showed improved EEG } \\
\text { activity recovery } \\
\text { b) } \mathrm{H}_{2} \text { fully or partially preserved } \\
\text { cerebrovascular reactivity and provided } \\
\text { modest neuroprotection }\end{array}$ & N/A \\
\hline Varga et al. (60) & $\mathrm{H}_{2}$ & Newborn piglets & $\begin{array}{l}\text { Asphyxia by tracheal occlusion } \\
\text { x } 8 \text { min followed by ventilation } \\
\text { with }\left(6 \% \mathrm{O}_{2}, 20 \% \mathrm{CO}_{2} \text { gas }\right. \\
\text { mixture } \times 20 \text { min }\end{array}$ & $\begin{array}{l}\text { a) } \mathrm{RA} \text { (room air) control group } \\
\text { b) Asphyxia+RA } \times 24 \mathrm{~h} \\
\text { c) Asphyxia+2.1\% } \mathrm{H}_{2} \times 4 \mathrm{~h} \text { then } \\
\mathrm{RA} \times 2 \mathrm{O} \mathrm{h}\end{array}$ & $\begin{array}{l}\mathrm{H}_{2} \text { treatment prohibited increase in COX-2 } \\
\text { build-up in neurons }\end{array}$ & N/A \\
\hline Domoki et al. (61) & $\mathrm{H}_{2}$ & Newborn piglets & $\begin{array}{l}\text { Asphyxia: Clamping } \\
\text { endotracheal tube in intubated } \\
\text { piglets } \times 10 \text { min. Both Sham } \\
\text { groups were ventilated in RA for } \\
10 \text { min. Following asphyxia, } \\
\text { animals were ventilated with } \\
\text { respective gases for } 4 \mathrm{~h} \text { then } \\
\text { euthanized }\end{array}$ & $\begin{array}{l}\text { a) Asphyxia/ventilation with RA } \\
\left(21 \% \mathrm{O}_{2}, 79 \% \mathrm{~N}_{2}\right) \text { ventilation } \\
\text { b) Asphyxia/ventilation with } \\
\mathrm{H}_{2}-\mathrm{RA}\left(2.1 \% \mathrm{H}_{2} ; 21 \% \mathrm{O}_{2} \text {; }\right. \\
\left.76.9 \% \mathrm{~N}_{2}\right) \text { ventilation } \\
\text { c) Time control group (Sham) } \\
\text { ventilated with } \mathrm{RA} \\
\text { d) Time control group (Sham) } \\
\text { ventilated with } \mathrm{H}_{2}-\mathrm{RA}\end{array}$ & $\begin{array}{l}\mathrm{H}_{2} \text {-RA ventilation: } \\
\text { a) Reduced brain injury in all brain areas } \\
\text { examined } \\
\text { b) Improved cerebrovascular reactivity to } \\
\text { hypercapnia but not to NMDA }\end{array}$ & N/A \\
\hline Linner et al. (62) & $\mathrm{O}_{2}$ & Newborn piglets & $\begin{array}{l}\text { Asphyxia in newborn piglets } \\
\text { (heart rate }<60 \mathrm{BMP} \text {, mean } \\
\text { arterial pressure }<30 \mathrm{mmHg} \text { ) }\end{array}$ & $\begin{array}{l}10 \text { min resuscitation with either: } \\
\text { a) } 1 \text { breath/min of } 100 \% \mathrm{O}_{2} \\
\text { b) } 1 \text { breath/min of air }\end{array}$ & $\begin{array}{l}\text { Piglets in oxygen group did not require } \\
\text { closed-chest cardiac massage (resuscitation) } \\
\text { All piglets in room air group required } \\
\text { closed-chest cardiac massage }\end{array}$ & $\mathrm{N} / \mathrm{A}$ \\
\hline Solas et al. (63) & $\mathrm{O}_{2}$ & Newborn piglets & $\begin{array}{l}\text { Hypoxia-ischemia-hypercapnia } \\
(\mathrm{HIH}) \text {. Hypoxia: breathing } 8 \% \mathrm{O}_{2} \\
\text { in } \mathrm{N}_{2} \\
\text { Ischemia: temporary bilateral } \\
\text { common carotid artery occlusion } \\
\text { Hypercapnia: mixing } \mathrm{CO}_{2} \text { with } \\
\text { inspiratory gas targeting }\left(\mathrm{PaCO}_{2}\right. \\
\text { 8-9 kPa) }\end{array}$ & $\begin{array}{l}\text { Two groups: } \\
\text { a) } \mathrm{HIH} 100 \% \text { : } \mathrm{HIH} \times 20 \text { min, } \\
\text { reperfusion, re-oxygenation } \\
\text { with } 100 \% \mathrm{O}_{2} \times 30 \text { min } \\
\text { followed by } 21 \% \mathrm{O}_{2} \times 90 \text { min } \\
\text { b) } \mathrm{HIH} 21 \% \text { : } \mathrm{HIH} \times 20 \text { min, } \\
\text { reperfusion, re-oxygenation } \\
\text { with } 21 \% \mathrm{O}_{2} \times 120 \text { min }\end{array}$ & $\begin{array}{l}\text { a) } \mathrm{HIH} 100 \% \text { had improved restoration of } \\
\text { mean arterial blood pressure } \\
\text { b) No difference in amino acid build up in } \\
\text { cerebral striatum (glutamate, taurine, and } \\
\text { alanine) between both groups }\end{array}$ & N/A \\
\hline
\end{tabular}


TABLE 2 | Continued

\begin{tabular}{|c|c|c|c|c|c|c|}
\hline References & Gas & Age species & Procedure & Intervention & Outcomes & Behavioral \\
\hline Solberg et al. (64) & $\mathrm{O}_{2}$ & Newborn piglets & $\begin{array}{l}\text { Hypoxia by inhaling } 8 \% \mathrm{O} 2 \text { in } \mathrm{N}_{2} \\
\text { until either the base excess } \\
\text { reaches } \sim 20 \mathrm{mM} \text { or the mean } \\
\text { arterial blood pressure }>15 \mathrm{~mm} \\
\mathrm{Hg}\end{array}$ & $\begin{array}{l}\text { Resuscitation } \times 30 \text { min with } \\
\text { a) } 21 \% \mathrm{O}_{2} \text { after hypoxia } \\
\text { b) } 40 \% \mathrm{O}_{2} \text { after hypoxia } \\
\text { c) } 100 \% \mathrm{O}_{2} \text { after hypoxia } \\
\text { d) } 100 \% \mathrm{O}_{2} \text { without hypoxia }\end{array}$ & $\begin{array}{l}\text { Resuscitation with } 100 \% \mathrm{O}_{2} \text { increased net } \\
\text { matrix metalloproteinase gelatinolytic activity in } \\
\text { corpus striatum, and caspase-3 expression } \\
\text { and activity compared to those resuscitated in } \\
21 \% \mathrm{O}_{2}\end{array}$ & $\mathrm{~N} / \mathrm{A}$ \\
\hline Kutzsche et al. (65) & $\mathrm{O}_{2}$ & Newborn piglets & $\begin{array}{l}\text { Hypoxia by inhaling } 8 \% \mathrm{O}_{2} \\
\times 20 \text { min }\end{array}$ & $\begin{array}{l}\text { Reoxygenation x } 30 \text { min with: } \\
\text { a) } 100 \% \mathrm{O}_{2} \\
\text { b) } 21 \% \mathrm{O}_{2} \text {, subdivided to } \\
\text { L-NAME vs. saline placebo }\end{array}$ & $\begin{array}{l}\text { L-NAME pretreatment decreased cerebral } \\
\text { perfusion and systemic blood pressure during } \\
\text { hypoxemia. Reoxygenation with } 100 \% \mathrm{O}_{2} \text { with } \\
\text { no adverse effect on cerebral perfusion. }\end{array}$ & $\mathrm{N} / \mathrm{A}$ \\
\hline Kutzsche et al. (66) & $\mathrm{O}_{2}$ & Newborn piglets & $\begin{array}{l}\text { Hypoxia by inhaling } 6 \% \mathrm{O}_{2} \\
\times 20 \text { min }\end{array}$ & $\begin{array}{l}\text { Reoxygenation } \times 30 \text { min with: } \\
\text { a) } 100 \% \mathrm{O}_{2} \\
\text { b) } 21 \% \mathrm{O}_{2}\end{array}$ & $\begin{array}{l}\text { a) Hypoxia decreased cerebral nitric oxide (NO) } \\
\text { concentration and decreased cerebral } \\
\text { perfusion } \\
\text { b) Both groups had restoration of NO } \\
\text { concentration with reperfusion. However, } \\
100 \% \mathrm{O}_{2} \text { group had a higher level } \\
\text { after reoxygenation }\end{array}$ & N/A \\
\hline Chakkarapani et al. (67) & $\mathrm{Xe}$ & Newborn piglets & $\begin{array}{l}\text { Hypoxia by inhaling } 5-7 \% \mathrm{O}_{2} \\
\text { until EEG background activity is } \\
\text { suppressed to }<7 \mathrm{uV}\end{array}$ & 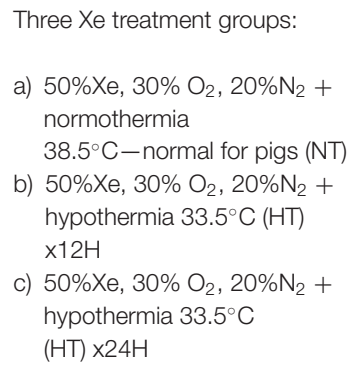 & $\begin{array}{l}\text { a) Xe had no effect on heart rate } \\
\text { b) Xe improved mean arterial blood pressure } \\
\text { c) No negative hemodynamic interaction } \\
\text { between Xe and hypothermia }\end{array}$ & $\mathrm{N} / \mathrm{A}$ \\
\hline Faulkner et al. (68) & $\mathrm{Xe}$ & Newborn piglets & $\begin{array}{l}\text { Occlusion of common carotid } \\
\text { arteries bilaterally and reducing } \\
\text { inspired } \mathrm{O}_{2} \text { to } 12 \% \times 10 \mathrm{~min}\end{array}$ & $\begin{array}{l}\text { Four groups: } \\
\text { a) Normothermia (NT) } \\
\text { b) } \mathrm{NT}+50 \% \mathrm{Xe} \times 24 \mathrm{H} \\
\text { c) Hypothermia } 33.5 \mathrm{C}(\mathrm{HT}) \\
\times 24 \mathrm{H} \\
\text { d) } \mathrm{HT}+50 \% \mathrm{Xe} \times 24 \mathrm{H}\end{array}$ & $\begin{array}{l}\text { a) Mean arterial blood pressure is lower in all } \\
\text { groups compared to NT } \\
\text { b) HTx } 24 \mathrm{H} \text { and } \mathrm{HT}+50 \% \mathrm{Xe} \times 24 \mathrm{H} \text { both } \\
\text { decreased cerebral magnetic resonance } \\
\text { spectroscopy abnormalities, nuclear DNA } \\
\text { fragmentation using TUNEL staining, and } \\
\text { caspase } 3 \text { activity in the cortex } \\
\text { c) Added Xe to HT did not reach statistical } \\
\text { significance of neuroprotection when } \\
\text { compared to HT alone }\end{array}$ & N/A \\
\hline
\end{tabular}

ANT, adenine nucleotide translocase; Ar, argon; ARE, antioxidant response elements; ATA, atmospheres absolute; Bax, Bcl2-associated X; Bcl-2, B-cell lymphoma 2; BCl-XL, B-cell lymphoma-extra-large; CAMP, cyclic adenosine 3',5'-monophosphate; $\mathrm{CO}_{2}$, carbon dioxide; HA, hydroxylamine; HBO, hyperbaric oxygen; He, helium; HI, hypoxia-ischemia; HIBD, hypoxic-ischemic brain damage; HO-1, heme oxygenase-1; HT, hypothermia; Keap1, kelch-like ECHassociated protein 1; LDH, lactate dehydrogenase; L-NAME, N(w)-nitro-L-arginine methyl ester; MCAO; middle cerebral artery occlusion; MDA, malondialdehyde; N/A, not applicable; N2, nitrogen gas; NF-KB, nuclear factor-kB; nNOS, neuronal nitric oxide synthase; N2O, nitrous oxide; NRF-2, nuclear factor erythroid 2-related factor 2; NT, normothermia; $\mathrm{O}_{2}$, oxygen gas; OGD, oxygen glucose deprivation; PARP, poly(ADP-ribose) polymerase; pCREB, phosphorylated CAMP response element binding protein; PI3K, phosphoinositide-3-kinase; RA, room air; SAM, S-adenosyl-L-methionine; TUNEL, terminal deoxynucleotidyl transferase dUTP nick end labeling; Tyr216-GSK-3 $\beta$, Phospho-glycogen synthase kinase-3 $\beta$ Tyr-216; Xe, xenon 


\section{Preclinical Studies}

In vitro, argon exposure led to improved neuronal cell survival after OGD and mechanical trauma (26). David et al. (25). showed that neuronal cells exposed to argon following OGD produced less LDH. Adult rats that underwent either transient cerebral ischemia for $60 \mathrm{~min}$ by middle cerebral artery occlusion (MCAO) or intrastriatal injection of NMDA were assessed for subcortical and cortical damage. Argon inhalation $1 \mathrm{~h}$ after NMDA injection to the right striatum significantly reduced cortical infarction size compared to the contralateral region. The maximum effect of argon inhalation was noted at a volume of $50 \%$. However, argon therapy after transient MCAO alleviated histopathologic cortical volume loss by $35 \%$ but increased subcortical volume loss by $35 \%$ (25). Adult rats treated with inhaled argon $1 \mathrm{~h}$ after transient MCAO demonstrated statistically significant reductions in infarct volumes at the levels of the cortex and basal ganglia without changes in the of mortality risk (27).

In vitro, exposure to $70 \%$ argon at $33^{\circ} \mathrm{C}$ for $2 \mathrm{~h}$ following OGD resulted in upregulation and subsequent increased expression of heme oxygenase-1 (HO-1), Bcl-2, and phospho-Akt in cortex and hypothalamus neuronal cell cultures (10). Conversely, phospho-glycogen synthase kinase-3 $\beta$ Tyr-216 (Tyr216-GSK$3 \beta$ ) expression was decreased. When the effects of HO-1 and phospho-Akt were blocked, argon's neuroprotective effect was lost at the cortical level, proving that neuroprotection was mediated by argon. In vivo, when Argon was combined with hypothermia for $2 \mathrm{~h}$ in neonatal rats following the RiceVannucci model of HI, the infarct volume and level of caspase-3 decreased. Although hypothermia alone had no positive effect on Nuclear Factor-kB (NF- $\kappa$ B), argon combined with hypothermia reduced the expression of NF- $\kappa \mathrm{B}$, which is a main inflammatory cascade component in the brain (10). In a piglet study argon combined with hypothermia preserved brain MRS (magnetic resonance spectroscopy) ATP, decreased MRS lactate/NAA peak, and expedited EEG background recovery when compared to hypothermia alone (58).

\section{Clinical Trials}

Currently, no one has performed a clinical trial of argon as a neuroprotective agent.

\section{Helium}

Helium is odorless, colorless, and tasteless. Heliox, a combination of $79 \%$ helium and $21 \%$ oxygen, which has lower viscosity than medical air has been used widely in patients with upper airway obstruction, to decrease work of breathing (80). In addition, it attenuates lung inflammation following acute lung injury of neonatal piglets (81). Preconditioning with helium protects the heart myocardium against HI (82). Helium was not shown to change cerebral blood flow in a human study (83).

\section{Preclinical Studies}

Infarct volumes and neurologic scores were assessed in adult rats that underwent left MCAO for $2 \mathrm{~h}$ followed by reperfusion for $1 \mathrm{~h}$. Adult male rats breathed $30 \%$ oxygen and $70 \%$ nitrogen (control group), 100\% oxygen (hyperoxia group), or $30 \%$ oxygen and $70 \%$ helium (heliox group). The infarct volume in the heliox group was smaller than the hyperoxia and control groups. Neurologic scores in the heliox group were significantly better compared to the control group (43). Neonatal pups preconditioned with helium $24 \mathrm{~h}$ prior to RiceVannucci model of HI for three 5-min periods demonstrated increased endogenous of nitric oxide production, increased the transcriptional factor Nrf2 activation and anti-oxidant enzyme expression, reduced brain infarct areas, and improved neurological testing scores when tested 2 weeks following injury. This suggests that the mechanism of neuroprotection of helium preconditioning is mediated by activation of antioxidase response element by NO (Supplementary Figure 1) (42). Similarly, inhaled helium (70\% balanced with oxygen for $90 \mathrm{~min}$ ) administered to neonatal rats following the RiceVannucci HI model increased neuronal cell survival but did not decrease infarct volume in prolonged HI (HI for 120 vs. $90 \mathrm{~min})(28)$.

\section{Clinical Trials}

No one has performed a clinical study with helium as a neuroprotective agent.

\section{Hydrogen}

Hydrogen is both an odorless and colorless gas. It has the lowest density of all gases and is by far the most abundant element in the universe, found on most stars and planets. It is primarily bound to oxygen as water with very scarce amounts in atmospheric air. Hydrogen is thought to protect against HI injury by binding free radicals formed by oxidative stress (i.e., hydroxyl radicals) $(41,84)$ and reducing cyclooxygenase-2 (COX-2) accumulation in the cerebral cortex (60).

\section{Preclinical Studies}

Adult mice that consumed hydrogen water had reduced oxidative stress in the hippocampus caused by chronic physical restraint (85). Inhaled hydrogen was also found to protect against intestinal (86) and myocardial ischemia in rats (87).

Hydrogen treatment for 30, 60, and 120 min decreased infarct size and brain apoptosis by reducing caspase- 3 and caspase- 12 activity in neonatal rats undergoing the Rice-Vannucci model of HI for 90 min (Supplementary Figure 1) (39). In a similar study that extended HI to 120 and $150 \mathrm{~min}$, neuroprotection was not shown (40). Furthermore, the latter study reported an increased infarct volume when rats were preconditioned with hydrogen more than 90 min prior to HI.

Newborn piglets subjected to asphyxia for $10 \mathrm{~min}$ followed by re-ventilation with hydrogen supplemented room air were shown to have ameliorated neuronal injury in the cortex, hippocampus, basal ganglia, cerebellum, and brain stem (61). Another study of newborn piglets treated with inhaled 2.1\% $\mathrm{H}_{2}$ after asphyxia by clamping the endotracheal tube for $8 \mathrm{~min}$ showed improved EEG activity recovery and preservation of cerebrovascular reactivity (59).

\section{Clinical Trials}

Currently hydrogen is not used for neuroprotection in ongoing or completed clinical studies. 


\section{Nitric Oxide}

NO produced by endothelial cells and acts as a potent vasodilator that is rapidly metabolized by oxyhemoglobin. Clinicians manage persistent pulmonary hypertension in neonates with inhaled NO (iNO) because it vasodilates the pulmonary vascular bed. Recent research showed that endogenous cardiac NO binds to plasma proteins and can affect distant organs such as the liver. This finding indicates that NO may have a broad effect in the human body (88). Inhaled nitric oxide had no effect on hemodynamics in rats treated with hypothermia following prolonged cardiopulmonary arrest (89).

\section{Preclinical Studies}

In vitro, OGD increases iron deposition and hydroxyl radical formation in neonatal brain hippocampal cell cultures. This process is mediated by endogenous production of NO. Neuronal cellular death is reduced by scavenging iron with deferoxamine and by blocking endogenous NO production by using L-NAME [N( $\omega)$-nitro-L-arginine methyl ester] (45).

On the contrary, exogenous administration of $50 \mathrm{ppm}$ iNO administered immediately after transient MCAO of adult mice was associated with selective dilation of arterioles in the ischemic areas. This dilation exclusively improved collateral blood flow and significantly reduced infarct size. Treatment with iNO for $24 \mathrm{~h}$ enhanced long-term adverse neurologic outcomes and survival in mice. This same study looked at perinatal asphyxia. Following the ligature of the left common carotid artery, neonatal pups were subjected to hypoxia for $50 \mathrm{~min}$ with or without $\mathrm{NO}$ (50 ppm) added to the gas mixture. Tissue loss was significantly attenuated 3 days after the insult (46).

$\mathrm{Li}$ et al. studied different concentrations and durations of iNO following transient MCAO for $1 \mathrm{~h}$ and reperfusion for 2 days. They found that the effect of iNO was dose-dependent and the maximum benefit of iNO occurred at $60 \mathrm{ppm}$ for 8 and $16 \mathrm{~h}$. Additionally, iNO administered for $8 \mathrm{~h}$ improved recovery from subarachnoid hemorrhage and reduced the inflammatory response (44).

Interestingly, one study by Zhu showed that inhaled nitric oxide provided neuroprotection only to male rats subjected to hypoxia-ischemia (47).

\section{Clinical Trials}

"Inhaled Nitric Oxide in Brain Injury" (NCT03260569) is an ongoing trial to evaluate the effect of $20 \mathrm{ppm}$ iNO on pulmonary mechanics in adults older than 18 years following traumatic brain injury.

\section{Carbon Dioxide}

Carbon dioxide $\left(\mathrm{CO}_{2}\right)$ is the fourth most common gas component in air following nitrogen, oxygen, and argon. In vivo, $\mathrm{CO}_{2}$ regulates min ventilation and cerebral blood flow. In the past few decades, overwhelming evidence has linked hypocapnia in preterm neonates to an increased risk of periventricular leukomalacia and intraventricular hemorrhage (90, 91). In contrast, permissive hypercapnia in preterm neonates is safe and reduces the length of mechanical ventilation for infants, which helps decrease the incidence of bronchopulmonary dysplasia (92).

\section{Preclinical Studies}

Vannucci et al. studied the relationship between various concentrations of $\mathrm{CO}_{2}$ and hypoxic-ischemic injury. They determined that pups subjected to $\mathrm{HI}$ without supplemental $\mathrm{CO}_{2}$ had a partial pressure of $\mathrm{CO}_{2}\left(\mathrm{PCO}_{2}\right)$ of $\sim 26 \mathrm{mmHg}$, and inhaling 3,6, and $9 \% \mathrm{CO}_{2}$ at the time of $\mathrm{HI}$ was associated with a $\mathrm{PCO}_{2}$ of 38,55 , and $71 \mathrm{mmHg}$, respectively (36). In a subsequent study, Vannucci et al. determined that extreme hypercapnia $\left(\mathrm{PCO}_{2} \sim 100 \mathrm{mmHg}\right)$ can be achieved by inhaling $15 \% \mathrm{CO}_{2}$ (37).

Based on the Rice-Vannucci model, hypocapnia in neonatal rats at the time of $\mathrm{HI}$ results in the most significant brain damage. Normocapnia $\left(3 \% \mathrm{CO}_{2}\right)$ produced less brain damage, while hypercapnia $\left(6 \% \mathrm{CO}_{2}\right)$ was associated with minimal or no brain damage based on histopathologic evaluation of cerebral hemisphere affected by hypoxic-ischemic injury via Rice-Vannucci model (36). Similarly, Kohzuki et al. found that brain injury was significantly ameliorated in neonatal rats treated with $6 \% \mathrm{CO} 2$ during $\mathrm{HI}$ for $30 \mathrm{~min}$ and staircase test scores improved compared to controls when tested at 30 days (35). Vannucci et al. determined that mild hypercapnia $\left(6 \% \mathrm{CO}_{2}\right)$ and normocapnia $\left(3 \% \mathrm{CO}_{2}\right)$ at the time of $\mathrm{HI}$ preserved cerebral blood flow in contrast to hypocapnia $\left(0 \% \mathrm{CO}_{2}\right)$, which reduced cerebral blood flow (38). Vannucci et al. found that neonatal rats exposed to extreme hypercapnia $\left(15 \% \mathrm{CO}_{2}\right)$ at the time of hypoxic-ischemic injury had significantly reduced cerebral blood flow and the greatest brain infarct size compared to normocapnic littermates (37).

\section{Clinical Trials}

"Hypoxic-Ischemic Encephalopathy Therapy Optimization in Neonates for Better Neuroprotection with Inhalative $\mathrm{CO}_{2}$ (HENRIC)" is a single-center, open-label, interventional trial (NCT02700854). The study is recruiting mechanically ventilated infants undergoing therapeutic hypothermia for HIE in Hungary. The study aim is to evaluate the safety and feasibility of a lowconcentration $\mathrm{CO}_{2}$ gas mixture $\left(5 \% \mathrm{CO}_{2}+95 \%\right.$ air $)$ with the hypothesis that hyperventilation driven by metabolic acidosis harms the brain.

\section{Carbon Monoxide}

Carbon monoxide (CO) is a colorless, odorless, and tasteless gas. It has an extremely high affinity to bind to hemoglobin and form carboxyhemoglobin. Compared to hemoglobin, carboxyhemoglobin has a significantly lower capacity to carry oxygen, which leads to cardio- and neurotoxicity (93).

\section{Preclinical Studies}

At high concentrations, $\mathrm{CO}$ is thought to be neurotoxic (29). However, lower levels of CO are neuroprotective (33). In vitro, $\mathrm{CO}$ upregulated $\mathrm{Bcl}-2$ in hippocampus cells (31). In addition, $\mathrm{CO}$ prevents oxidative stress-induced cortical astrocyte apoptosis by preventing dissipation of mitochondrial membrane potential, caspase 3 activation, and cytochrome c 
release (30). At the mitochondrial level, $\mathrm{CO}$ prevents membrane depolarization induced by calcium and inhibits mitochondrial swelling. It also prevents membrane pore formation by modulating adenine nucleotide translocase (ANT) activity (30). ANT plays an essential rule in the stability of the mitochondrial inner membrane. It is influenced by anti- and proapoptotic agents (i.e., Bcl family) leading to inhibition or activation of pore formation in the mitochondrial membrane, respectively (94).

Electrical acupuncture into rat brains subjected to hypoxicischemic injury leads to increased intrinsic CO levels via overexpression of heme oxygenase $1(\mathrm{HO}-1)$ in the cortex and was associated with decreased cortical injury (34). Exogenous $\mathrm{CO}$ mimics the neuroprotective effects of HO-1 by reducing autoimmune neuroinflammation (95). CO causes upregulation of HO-1 by promoting cytoplasmic dissociation of Nrf2 from Keap1. This dissociation leads Nrf2 to translocate to the nucleus and bind to the antioxidant response elements (ARE) (Supplementary Figure 1) (32).

Inhalation of 125 and $250 \mathrm{ppm}$ of $\mathrm{CO}$ for $18 \mathrm{~h}$ following $90 \mathrm{~min}$ of transient MCAO and $48 \mathrm{~h}$ of reperfusion was associated with smaller infarct size and reduced brain edema (33). NRF2 knockout adult mice lost CO neuroprotection following permanent MCAO (32).

\section{Clinical Trials}

Currently, $\mathrm{CO}$ for neuroprotection is not being investigated in clinical studies.

\section{Oxygen}

Oxygen is the third most common element in the universe and is considered the cornerstone of life. Water in plants and algae produce oxygen, which mitochondria use to produce ATP. Reactive oxygen species (ROS) like superoxide are formed by a partial reduction of oxygen during cellular metabolism and are exaggerated by cellular injury such as hypoxicischemic injury.

In 2011, AAP changed the neonatal resuscitation program (NRP) guidelines to resuscitate term infants with room air instead of $100 \%$ based on two metanalysis studies showing less mortality in infants in room air $(96,97)$. This change was adopted after major therapeutic hypothermia for HIE trials were completed raising the question as whether resuscitation with supplemental oxygen may affect hypothermic neuroprotection. Dalen et al. showed that resuscitation with $100 \%$ oxygen negated the neuroprotective benefits of hypothermia in neonatal rats subjected to HI (49).

\section{Preclinical Studies}

The evidence regarding resuscitation with $100 \%$ oxygen is conflicting. A recent study done by Smit et al. failed to show statistical difference in cortical and hippocampal volume loss or in short-term neurological testing between immature rats resuscitated with either 21 or $100 \%$ oxygen following a modified
Rice-Vannucci model of hypoxic-ischemic brain injury (50). Newborn piglets resuscitated with $100 \% \mathrm{O}_{2}$ improved restoration of mean arterial blood pressure with no difference in amino acid build up in cerebral striatum (glutamate, taurine, and alanine) when compared to those resuscitated with $21 \% \mathrm{O}_{2}$ (63). Reoxygenation of asphyxiated piglets with $100 \% \mathrm{O}_{2}$ did not change cerebral perfusion but restored nitric oxide level in the cortex to a higher level compared to those resuscitated with $21 \%$ $\mathrm{O}_{2}(65,66)$.

\section{Clinical Studies}

"Hyperbaric Oxygen Therapy Improves Outcome of HypoxicIschemic Encephalopathy" (NCT02894866) is an ongoing multicenter randomized international study that is still recruiting patients. The study aim is to evaluate the safety and efficacy of hyperbaric oxygen in term infants with HIE.

\section{CONCLUSION}

HIE management remains a challenge. Although therapeutic hypothermia has improved survival in infants with HIE, 7 infants needed to be treated to prevent death or major neurodevelopmental disability. Preclinical studies show that inhaled gases are a promising adjunct therapy that could improve outcomes. Of the reviewed inhaled gases and pending the results of the CoolXenon3 Trial, xenon appears to be the most promising for clinical use. Several of the other reviewed gases such as $\mathrm{NO}$ and $\mathrm{C}$ possess anti-inflammatory properties via the Nrf2 pathway and neuroprotection promise in preclinical settings. Further clinical trials should focus on proving the efficacy and safety of inhaled gases in the clinical setting.

\section{AUTHOR CONTRIBUTIONS}

MW and SD contributed conception and design of the study. SS, HG, and ME searched literature and organized the database. YT wrote the first draft of the manuscript. MW wrote sections of the manuscript. All authors contributed to manuscript revision, read and approved the submitted version.

\section{FUNDING}

This work was supported in part by a grant from the McKnight Brain Research Foundation, Brain and Spinal Cord Injury Research Trust Fund (SD, MW), and by the National Institute of Health (NIH), NS103036 (SD).

\section{SUPPLEMENTARY MATERIAL}

The Supplementary Material for this article can be found online at: https://www.frontiersin.org/articles/10.3389/fped. 2019.00558/full\#supplementary-material 


\section{REFERENCES}

1. Kurinczuk JJ, White-Koning M, Badawi N. Epidemiology of neonatal encephalopathy and hypoxic-ischaemic encephalopathy. Early Hum Dev. (2010) 86:329-38. doi: 10.1016/j.earlhumdev.2010.05.010

2. Azzopardi DV, Strohm B, Edwards AD, Dyet L, Halliday HL, Juszczak E, et al. Moderate hypothermia to treat perinatal asphyxial encephalopathy. $N$ Engl J Med. (2009) 361:1349-58. doi: 10.1056/NEJMoa0900854

3. Gluckman PD, Wyatt JS, Azzopardi D, Ballard R, Edwards AD, Ferriero DM, et al. Selective head cooling with mild systemic hypothermia after neonatal encephalopathy: multicentre randomised trial. Lancet. (2005) 365:663-70. doi: 10.1016/S0140-6736(05)70932-6

4. Shankaran S, Laptook AR, Ehrenkranz RA, Tyson JE, McDonald SA, Donovan EF, et al. Whole-body hypothermia for neonates with hypoxic-ischemic encephalopathy. N Engl J Med. (2005) 353:1574-84. doi: 10.1056/NEJMcps050929

5. Tagin MA, Woolcott CG, Vincer MJ, Whyte RK, Stinson DA. Hypothermia for neonatal hypoxic ischemic encephalopathy. Arch Pediatr Adolesc Med. (2012) 166:558-66. doi: 10.1001/archpediatrics.2011.1772

6. Shankaran S, Laptook AR, Pappas A, McDonald SA, Das A, Tyson JE, et al. Effect of depth and duration of cooling on deaths in the NICU among neonates with hypoxic ischemic encephalopathy a randomized clinical trial. J Am Med Assoc. (2014) 312:2629-39. doi: 10.1001/jama.2014. 16058

7. Shankaran S, Laptook AR, Pappas A, McDonald SA, Das A, Tyson JE, et al. Effect of depth and duration of cooling on death or disability at age 18 months among neonates with hypoxic-ischemic encephalopathy a randomized clinical trial. J Am Med Assoc. (2017) 318:57-67. doi: 10.1001/jama.2017.7218

8. Lynch C, Baum J, Tenbrinck R, Weiskopf RB. Xenon anesthesia. Anesthesiol J Am Soc Anesthesiol. (2000) 92:865-70. doi: 10.1097/00000542-200003000-00031

9. Esencan E, Yuksel S, Tosun YB, Robinot A, Solaroglu I, Zhang J. XENON in medical area: emphasis on neuroprotection in hypoxia and anesthesia. Med Gas Res. (2013) 3:4. doi: 10.1186/2045-9912-3-4

10. Zhao H, Mitchell S, Koumpa S, Cui YT, Lian Q, Hagberg H, et al. Heme oxygenase-1 mediates neuroprotection conferred by argon in combination with hypothermia in neonatal hypoxia-ischemia brain injury. Anesthesiology. (2016) 125:180-92. doi: 10.1097/ALN.0000000000001128

11. Thoresen M, Hobbs CE, Wood T, Chakkarapani E, Dingley J. Cooling combined with immediate or delayed xenon inhalation provides equivalent long-term neuroprotection after neonatal hypoxia-ischemia. J Cereb Blood Flow Metab. (2009) 29:707-14. doi: 10.1038/jcbfm.2008.163

12. Hobbs C, Thoresen M, Tucker A, Aquilina K, Chakkarapani E, Dingley J. Xenon and hypothermia combine additively, offering long-term functional and histopathologic neuroprotection after neonatal hypoxia/ischemia. Stroke. (2008) 39:1307-13. doi: 10.1161/STROKEAHA.107.499822

13. Martin JL, Ma D, Hossain M, Xu J, Sanders RD, Franks NP, et al. Asynchronous administration of xenon and hypothermia significantly reduces brain infarction in the neonatal rat. Br J Anaesth. (2007) 98:236-40. doi: 10.1093/bja/ael340

14. Sabir H, Osredkar D, Maes E, Wood T, Thoresen M. Xenon combined with therapeutic hypothermia is not neuroprotective after severe hypoxia-ischemia in neonatal rats. PLoS ONE. (2016) 11:e156759. doi: 10.1371/journal.pone.0156759

15. Azzopardi D, Robertson NJ, Bainbridge A, Cady E, Charles-EdwardsG, Deierl A, et al. Moderate hypothermia within $6 \mathrm{~h}$ of birth plus inhaled xenon versus moderate hypothermia alone after birth asphyxia (TOBY-Xe): a proofof-concept, open-label, randomised controlled trial. Lancet Neurol. (2016) 15:145-53. doi: 10.1016/S1474-4422(15)00347-6

16. Wassink G, Gunn ER, Drury PP, Bennet L, Gunn AJ. The mechanisms and treatment of asphyxial encephalopathy. Front Neurosci. (2014) 8:40. doi: $10.3389 /$ fnins.2014.00040

17. Ferriero DM. Neonatal brain injury. N. Engl. J. Med. (2004) 351:1985-95. doi: 10.1056/NEJMra041996

18. Bennet L, Roelfsema V, Pathipati P, Quaedackers JS, Gunn AJ. Relationship between evolving epileptiform activity and delayed loss of mitochondrial activity after asphyxia measured by near-infrared spectroscopy in preterm fetal sheep. J Physiol. (2006) 572:141-54. doi: 10.1113/jphysiol.2006.105197
19. Gunn AJ, Gunn TR, De Haan HH, Williams CE, Gluckman PD. Dramatic neuronal rescue with prolonged selective head cooling after ischemia in fetal lambs. J Clin Invest. (1997) 99:248-56. doi: 10.1172/JCI119153

20. Bennet L, Tan S, Van Den Heuij L, Derrick M, Groenendaal F, van Bel F, et al. Cell therapy for neonatal hypoxia-ischemia and cerebral palsy. Ann Neurol. (2012) 71:589-600. doi: 10.1002/ana.22670

21. Schmitt KRL, Diestel A, Lehnardt S, Schwartlander R, Lange PE, Berger F, et al. Hypothermia suppresses inflammation via ERK signaling pathway in stimulated microglial cells. J Neuroimmunol. (2007) 189:7-16. doi: 10.1016/j.jneuroim.2007.06.010

22. Seo J-W, Kim J-H, Kim J-H, Seo M, Han HS, Park J, et al. Timedependent effects of hypothermia on microglial activation and migration. $J$ Neuroinflammation. (2012) 9:694. doi: 10.1186/1742-2094-9-164

23. Bossenmeyer-Pourié C, Koziel V, Daval J-L. Effects of hypothermia on hypoxia-induced apoptosis in cultured neurons from developing rat forebrain: comparison with preconditioning. Pediatr Res. (2000) 47:385-91. doi: 10.1203/00006450-200003000-00017

24. Ohmura A, Nakajima W, Ishida A, Yasuoka N, Kawamura M, Miura S, et al. Prolonged hypothermia protects neonatal rat brain against hypoxic-ischemia by reducing both apoptosis and necrosis. Brain Dev. (2005) 27:517-26. doi: 10.1016/j.braindev.2005.01.004

25. David HN, Haelewyn B, Degoulet M, Colomb DG, Risso JJ, Abraini $\mathrm{JH}$. Ex vivo and in vivo neuroprotection induced by argon when given after an excitotoxic or ischemic insult. PLoS ONE. (2012) 7:e30934. doi: 10.1371/journal.pone.0030934

26. Loetscher PD, Rossaint J, Rossaint R, Weis J, Fries M, Fahlenkamp A, et al. Argon: neuroprotection in in vitro models of cerebral ischemia and traumatic brain injury. Crit Care. (2009) 13:R206. doi: 10.1186/cc8214

27. Ryang Y-M, Fahlenkamp AV, Rossaint R, Wesp D, Loetscher PD, Beyer C, et al. Neuroprotective effects of argon in an in vivo model of transient middle cerebral artery occlusion in rats*. Crit Care Med. (2011) 39:1448-53. doi: 10.1097/CCM.0b013e31821209be

28. Zhuang L, Yang T, Zhao H, Fidalgo AR, Vizcaychipi MP, Sanders $\mathrm{RD}$, et al. The protective profile of argon, helium, and xenon in a model of neonatal asphyxia in rats*. Crit Care Med. (2012) 40:1724-30. doi: 10.1097/CCM.0b013e3182452164

29. Cheng Y, Thomas A, Mardini F, Bianchi SL, Tang JX, Peng J, et al. Neurodevelopmental consequences of sub-clinical carbon monoxide exposure in newborn mice. PLoS ONE. (2012) 7:e32029. doi: 10.1371/journal.pone.0032029

30. Queiroga CSF, Almeida AS, Martel C, Brenner C, Alves PM, Vieira HLA. Glutathionylation of adenine nucleotide translocase induced by carbon monoxide prevents mitochondrial membrane permeabilization and apoptosis. J Biol Chem. (2010) 285:17077-88. doi: 10.1074/jbc.M109.065052

31. Queiroga CSF, Tomasi S, Widerøe M, Alves PM, Vercelli A, Vieira HLA. Preconditioning triggered by carbon monoxide (CO) provides neuronal protection following perinatal hypoxia-ischemia. PLoS ONE. (2012) 7:e42632. doi: 10.1371 /journal.pone. 0042632

32. Wang B, Cao W, Biswal S, Doré S. Carbon monoxide-activated Nrf2 pathway leads to protection against permanent focal cerebral ischemia. Stroke. (2011) 42:2605-10. doi: 10.1161/STROKEAHA.110.607101

33. Zeynalov E, Doré S. Low doses of carbon monoxide protect against experimental focal brain ischemia. Neurotox Res. (2009) 15:133-7. doi: 10.1007/s12640-009-9014-4

34. Liu Y, Li Z, Shi X, Liu Y, Li W, Duan G, et al. Neuroprotection of up-regulated carbon monoxide by electrical acupuncture on perinatal hypoxic-ischemic brain damage in rats. Neurochem Res. (2014) 39:1724-32. doi: 10.1007/s11064-014-1366-3

35. Kohzuki M, Tomimatsu T, Fukuda H, Kanagawa T, Kanzaki T, Shimoya K, et al. Long-term neuroprotective effects of carbon dioxide on neonatal rat hypoxic-ischemic brain injury: an experimental study of skilled motor tasks. Am J Obstet Gynecol. (2006) 195:240-5. doi: 10.1016/j.ajog.2005.12.036

36. Vannucci R, Towfigi J, Heitijan D, Brucklacher R. Carbon dioxide protects the perinatal brain from hypoxic-ischemic damage: an experimental study in the immature rat. Pediatrics. (1995) 95:868-74.

37. Vannucci RC, Towfighi J, Brucklacher RM, Vannucci SJ. Effect of extreme hypercapnia on hypoxic-ischemic brain damage in the immature rat. Pediatr Res. (2001) 49:799-803. doi: 10.1203/00006450-200106000-00015 
38. Vannucci RC, Brucklacher RM, Vannucci SJ. Effect of carbon dioxide on cerebral metabolism during hypoxia-ischemia in the immature rat. Pediatr Res. (1997) 42:24-9. doi: 10.1203/00006450-199707000-00005

39. Cai J, Kang Z, Liu WW, Luo X, Qiang S, Zhang JH, et al. Hydrogen therapy reduces apoptosis in neonatal hypoxia-ischemia rat model. Neurosci Lett. (2008) 441:167-72. doi: 10.1016/j.neulet.2008.05.077

40. Matchett GA, Fathali N, Hasegawa Y, Jadhav V, Ostrowski RP, Martin RD, et al. Hydrogen gas is ineffective in moderate and severe neonatal hypoxia-ischemia rat models. Brain Res. (2009) 1259:90-7. doi: 10.1016/j.brainres.2008.12.066

41. Ohsawa I, Ishikawa M, Takahashi K, Watanabe M, Nishimaki K, Yamagata $\mathrm{K}$, et al. Hydrogen acts as a therapeutic antioxidant by selectively reducing cytotoxic oxygen radicals. Nat Med. (2007) 13:688-94. doi: 10.1038/nm1577

42. Li Y, Liu K, Kang ZM, Sun XJ, Liu WW, Mao YF. Helium preconditioning protects against neonatal hypoxia-ischemia via nitric oxide mediated upregulation of antioxidases in a rat model. Behav Brain Res. (2016) 300:31-7. doi: 10.1016/j.bbr.2015.12.001

43. Pan Y, Zhang H, VanDeripe DR, Cruz-Flores S, Panneton WM. Heliox and oxygen reduce infarct volume in a rat model of focal ischemia. Exp Neurol. (2007) 205:587-90. doi: 10.1016/j.expneurol.2007.03.023

44. Li YS, Shemmer B, Stone E, A Nardi M, Jonas S, Quartermain D. Neuroprotection by inhaled nitric oxide in a murine stroke model is concentration and duration dependent. Brain Res. (2013) 1507:134-45. doi: 10.1016/j.brainres.2013.02.031

45. Lu Q, Harris VA, Rafikov R, Sun X, Kumar S, Black SM. Nitric oxide induces hypoxia ischemic injury in the neonatal brain via the disruption of neuronal iron metabolism. Redox Biol. (2015) 6:112-21. doi: 10.1016/j.redox.2015.06.007

46. Terpolilli NA, Kim SW, Thal SC, Kataoka H, Zeisig V, Nitzsche B, et al. Inhalation of nitric oxide prevents ischemic brain damage in experimental stroke by selective dilatation of collateral arterioles. Circ Res. (2012) 110:72738. doi: 10.1161/CIRCRESAHA.111.253419

47. Zhu C, Sun Y, Gao J, Wang X, Plesnila N, Blomgren K. Inhaled nitric oxide protects males but not females from neonatal mouse hypoxia-ischemia brain injury. Transl Stroke Res. (2013) 4:201-7. doi: 10.1007/s12975-012-0217-2

48. Calvert JW, Zhou C, Nanda A, Zhang JH. Effect of hyperbaric oxygen on apoptosis in neonatal hypoxia-ischemia rat model. J Appl Physiol. (2003) 95:2072-80. doi: 10.1152/japplphysiol.00630.2003

49. Dalen ML, Liu X, Elstad M, Løberg EM, Saugstad OD, Rootwelt T, et al. Resuscitation with $100 \%$ oxygen increases injury and counteracts the neuroprotective effect of therapeutic hypothermia in the neonatal rat. Pediatr Res. (2012) 71:247-52. doi: 10.1038/pr.2011.43

50. Smit E, Liu X, Gill H, Jary S, Wood T, Thoresen M. The effect of resuscitation in $100 \%$ oxygen on brain injury in a newborn rat model of severe hypoxic-ischaemic encephalopathy. Resuscitation. (2015) 96:214-9. doi: 10.1016/j.resuscitation.2015.07.050

51. Dingley J, Tooley J, Liu X, Scull-Brown E, Elstad M, Chakkarapani E, et al. Xenon ventilation during therapeutic hypothermia in neonatal encephalopathy: a feasibility study. Pediatrics. (2014) 133:809-18. doi: 10.1542/peds.2013-0787

52. Luo Y, Ma D, Ieong E, Sanders RD, Yu B, Hossain M, et al. Xenon and sevoflurane protect against brain injury in a neonatal asphyxia model. Anesthesiology. (2008) 109:782-9. doi: 10.1097/ALN.0b013e3181895f88

53. Ma D, Lim T, Xu J, Tang H, Wan Y, Zhao H, et al. Xenon preconditioning protects against renal ischemic-reperfusion injury via HIF-1 activation. J Am Soc Nephrol. (2009) 20:713-20. doi: 10.1681/ASN.2008070712

54. Ma D, Hossain M, Pettet GK, Luo Y, Lim T, Akimov S, et al. Xenon preconditioning reduces brain damage from neonatal asphyxia in rats. J Cereb Blood Flow Metab. (2006) 26:199-208. doi: 10.1038/sj.jcbfm.9600184

55. Sabir H, Walløe L, Dingley J, Smit E, Liu X, Thoresen M. Combined treatment of Xenon and hypothermia in newborn rats - Additive or synergistic effect? PLoS ONE. (2014) 9:e109845. doi: 10.1371/journal.pone.0109845

56. Yang T, Zhuang L, Rei Fidalgoo AM, Petrides E, Terrando N, Wu X, et al. Xenon and sevoflurane provide analgesia during labor and fetal brain protection in a perinatal rat model of hypoxia-ischemia. PLOS ONE. (2012) 7:e37020. doi: 10.1371/journal.pone.0037020

57. Alderliesten T, Favie LMA, Neijzen RW, Auwärter V, Nijboer CH, Marges RE, et al. Neuroprotection by argon ventilation after perinatal asphyxia: a safety study in newborn piglets. PLoS ONE. (2014) 9:e113575. doi: 10.1371/journal.pone.0113575

58. Broad KD, Fierens I, Fleiss B, Rocha-Ferreira E, Ezzati M, Hassell J, et al. Inhaled $45-50 \%$ argon augments hypothermic brain protection in a piglet model of perinatal asphyxia. Neurobiol Dis. (2016) 87:29-38. doi: 10.1016/j.nbd.2015.12.001

59. Oláh O, Tóth-Szki V, Temesvári P, Bari F, Domoki F. Delayed neurovascular dysfunction is alleviated by hydrogen in asphyxiated newborn pigs. Neonatology. (2013) 104:79-86. doi: 10.1159/000348445

60. Varga V, Németh J, Oláh O, Tóth-Szuki V, Kovács V, Remzso G, et al. Molecular hydrogen alleviates asphyxia-induced neuronal cyclooxygenase2 expression in newborn pigs. Acta Pharmacol Sin. (2018) 39:1273-83. doi: 10.1038/aps.2017.148

61. Domoki F, Oláh O, Zimmermann A, Németh I, Tóth-Szuki V, Hugyecz $\mathrm{M}$, et al. Hydrogen is neuroprotective and preserves cerebrovascular reactivity in asphyxiated newborn pigs. Pediatr Res. (2010) 68:1. doi: 10.1203/PDR.0b013e3181f2e81c

62. Linner R, Cunha-Goncalves D, Perez-de-Sa V. One oxygen breath shortened the time to return of spontaneous circulation in severely asphyxiated piglets. Acta Paediatr Int J Paediatr. (2017) 106:1556-63. doi: 10.1111/apa. 13920

63. Solås AB, Kalous P, Saugstad OD. Reoxygenation with 100 or $21 \%$ oxygen after cerebral hypoxemia-ischemia- hypercapnia in newborn piglets. Biol Neonate. (2004) 85:105-11. doi: 10.1159/000074966

64. Solberg R, Løberg EM, Andresen JH, Wright MS, Charrat E, Khrestchatisky $\mathrm{M}$, et al. Resuscitation of newborn piglets. Short-term influence of $\mathrm{FiO} 2$ on matrix metalloproteinases, caspase-3 and BDNF. PLoS ONE. (2010) 5:e14261. doi: 10.1371/journal.pone.0014261

65. Kutzsche S, Solas AB, Lyberg T, Saugstad OD. Nitric oxide synthesis inhibition during cerebral hypoxemia and reoxygenation with $100 \%$ oxygen in newborn pigs. Biol Neonate. (2002) 82:197-206. doi: 10.1159/000063613

66. Kutzsche S, Kirkeby OJ, Rise IR, Saugstad OD. Effects of hypoxia and reoxygenation with $21 \%$ and $100 \%$-oxygen on cerebral nitric oxide concentration and microcirculation in newborn piglets. Biol Neonate. (1999) 76:153-67. doi: 10.1159/000014155

67. Chakkarapani E, Thoresen M, Liu X, Walloe L, Dingley J. Xenon offers stable haemodynamics independent of induced hypothermia after hypoxia-ischaemia in newborn pigs. Intensive Care Med. (2012) 38:316-23. doi: 10.1007/s00134-011-2442-7

68. Faulkner S, Bainbridge A, Kato T, Chandrasekaran M, Kapetanakis AB, Hristova $\mathrm{M}$, et al. Xenon augmented hypothermia reduces early lactate/Nacetylaspartate and cell death in perinatal asphyxia. Ann Neurol. (2011) 70:133-50. doi: 10.1002/ana.22387

69. Hofland J, Ouattara A, Fellahi J-L, Gruenewald M, Hazebroucq J, Ecoffey $\mathrm{C}$, et al. Effect of Xenon anesthesia compared to sevoflurane and total intravenous anesthesia for coronary artery bypass graft surgery on postoperative cardiac troponin release. Anesthesiology. (2017) 127:918-33. doi: 10.1097/ALN.0000000000001873

70. Preckel B, Müllenheim J, Moloschavij A, Thämer V, Schlack W. Xenon administration during early reperfusion reduces infarct size after regional ischemia in the rabbit heart in vivo. Anesth Analg. (2000) 91:1327-32. doi: 10.1097/00000539-200012000-00003

71. Baumert JH, Hein M, Gerets C, Baltus T, Hecker KE, Rossaint R. The effect of xenon anesthesia on the size of experimental myocardial infarction. Anesth Analg. (2007) 105:1200-6. doi: 10.1213/01.ane.0000284697.73471.9c

72. Franks NP, Dickinson R, De Sousa SLM, Hall AC, Lieb WR. How does xenon produce anaesthesia? Nature. (1998) 396:324. doi: 10.1038/24525

73. Natale G, Cattano D, Abramo A, Forfori F, Fulceri F, Fornai F, et al. Morphological evidence that xenon neuroprotects against N-methyl-DLaspartic acid-induced damage in the rat arcuate nucleus: a time-dependent study. Ann N Y Acad Sci. (2006) 1074:650-8. doi: 10.1196/annals.1369.063

74. Dinse A, Föhr KJ, Georgieff M, Beyer C, Bulling A, Weigt HU. Xenon reduces glutamate-, AMPA-, and kainate-induced membrane currents in cortical neurones. Br J Anaesth. (2005) 94:479-85. doi: 10.1093/bja/aei080

75. Petzelt C. Participation of the $\mathrm{Ca} 2+$ calmodulin-activated kinase II in the control of metaphase-anaphase transition in human cells. Cell Biol Int. (2001) 25:403-9. doi: 10.1006/cbir.2000.0646 
76. Dingley J, Tooley J, Porter H, Thoresen M. Xenon provides short-term neuroprotection in neonatal rats when administered after hypoxia-ischemia. Stroke. (2006) 37:501-6. doi: 10.1161/01.STR.0000198867.31134.ac

77. Wilson BE, Mochon E, Boxer LM. Induction of bcl-2 expression by phosphorylated CREB proteins during B-cell activation and rescue from apoptosis. Mol Cell Biol. (1996) 16:5546-56. doi: 10.1128/MCB.16.10.5546

78. Azzopardi D, Robertson NJ, Kapetanakis A, Griffiths J, Rennie JM, Mathieson SR, et al. Anticonvulsant effect of xenon on neonatal asphyxial seizures. Arch Dis Child Fetal Neonatal Ed. (2013) 98:F437-9. doi: 10.1136/archdischild-2013-303786

79. Abraini JH, Kriem B, Balon N, Rostain J-C, Risso J-J. Gamma-aminobutyric acid neuropharmacological investigations on narcosis produced by nitrogen, argon, or nitrous oxide. Anesth Analg. (2003) 96:746-9. doi: 10.1213/01.ANE.0000050282.14291.38

80. McGarvey JM, Pollack CV. Heliox in airway management. Emerg Med Clin North Am. (2008) 26:905-20. doi: 10.1016/j.emc.2008.07.007

81. Nawab US, Touch SM, Irwin-Sherman T, Blackson TJ, Greenspan JS, Zhu G, et al. Heliox attenuates lung inflammation and structural alterations in acute lung injury. Pediatr Pulmonol. (2005) 40:524-32. doi: 10.1002/ppul.20304

82. Heinen A, Huhn R, Smeele KMA, Zuurbier CJ, Schlack W, Preckel B, et al. Helium-induced Preconditioning in young and old rat heart. Anesthesiology. (2008) 109:830-6. doi: 10.1097/ALN.0b013e3181895aa0

83. Zinkstok SM, Bertens D, De Kruijk JR, Tromp SC. Effect of helium on cerebral blood flow: a $\mathrm{n}=1$ trial in a healthy young person. Perspect Med. (2012) 1-12:301-3. doi: 10.1016/j.permed.2012.02.009

84. Fukuda K, Asoh S, Ishikawa M, Yamamoto Y, Ohsawa I, Ohta S. Inhalation of hydrogen gas suppresses hepatic injury caused by ischemia/reperfusion through reducing oxidative stress. Biochem Biophys Res Commun. (2007) 361:670-4. doi: 10.1016/j.bbrc.2007.07.088

85. Nagata K, Nakashima-Kamimura N, Mikami T, Ohsawa I, Ohta S. Consumption of molecular hydrogen prevents the stress-induced impairments in hippocampus-dependent learning tasks during chronic physical restraint in mice. Neuropsychopharmacology. (2009) 34:501-8. doi: $10.1038 /$ npp. 2008.95

86. Buchholz BM, Kaczorowski DJ, Sugimoto R, Yang R, Wang Y, Billiar TR, et al. Hydrogen inhalation ameliorates oxidative stress in transplantation induced intestinal graft injury. Am J Transplant. (2008) 8:2015-24. doi: 10.1111/j.1600-6143.2008.02359.x

87. Hayashida K, Sano M, Ohsawa I, Shinmura K, Tamaki K, Kimura K, et al. Inhalation of hydrogen gas reduces infarct size in the rat model of myocardial ischemia-reperfusion injury. Biochem Biophys Res Commun. (2008) 373:30-5. doi: 10.1016/j.bbrc.2008.05.165

88. Elrod JW, Calvert JW, Gundewar S, Bryan NS, Lefer DJ. Nitric oxide promotes distant organ protection: evidence for an endocrine role of nitric oxide. Proc Natl Acad Sci USA. (2008) 105:11430-5. doi: 10.1073/pnas.0800700105
89. Brücken A, Bleilevens C, Berger P, Nolte K, Gaisa NT, Rossaint R, et al. Effects of inhaled nitric oxide on outcome after prolonged cardiac arrest in mild therapeutic hypothermia treated rats. Sci Rep. (2018) 8:6743. doi: 10.1038/s41598-018-25213-1

90. Greisen G, Munck H, Lou H. Severe hypocarbia in preterm infants and neurodevelopmental deficit. Acta Paediatr Scand. (1987) 76:401-4. doi: 10.1111/j.1651-2227.1987.tb10489.x

91. Graziani LJ, Spitzer AR, Mitchell DG, Merton DA, Stanley C, Robinson $\mathrm{N}$, et al. Mechanical ventilation in preterm infants: neurosonographic and developmental studies. Pediatrics. (1992) 90:515-22.

92. Mariani G, Cifuentes J, Carlo WA. Randomized trial of permissive hypercapnia in preterm infants. Pediatrics. (1999) 104:1082-8. doi: $10.1542 /$ peds.104.5.1082

93. Chu K, Jung KH, Kim HJ, Jeong SW, Kang DW, Roh JK. Diffusion-weighted MRI and 99mTc-HMPAO SPECT in delayed relapsing type of carbon monoxide poisoning: evidence of delayed cytotoxic edema. Eur Neurol. (2004) 51:98-103. doi: 10.1159/000076536

94. Belzacq AS, Vieira HLA, Verrier F, Vandecasteele G, Cohen I, Prévost MC, et al. Bcl-2 and Bax modulate adenine nucleotide translocase activity. Cancer Res. (2003) 63:541-6.

95. Chora ÂA, Fontoura P, Cunha A, Pais TF, Cardoso S, Ho PP, et al. Heme oxygenase-1 and carbon monoxide suppress autoimmune neuroinflammation. J Clin Invest. (2007) 117:3-12. doi: 10.1172/JCI 28844

96. Davis PG, Tan A, O'Donnell CPF, Schulze PA. Resuscitation of newborn infants with $100 \%$ oxygen or air: a systematic review and meta-analysis. Lancet. (2004) 364:1329-33. doi: 10.1016/S0140-6736(04)17189-4

97. Rabi Y, Rabi D, Yee W. Room air resuscitation of the depressed newborn: a systematic review and meta-analysis. Resuscitation. (2007) 72:353-63. doi: 10.1016/j.resuscitation.2006.06.134

Disclosure: Mallinckrodt Pharmaceuticals provided nitric oxide gas for a preclinical study in which all authors are involved.

Conflict of Interest: The authors declare that the research was conducted in the absence of any commercial or financial relationships that could be construed as a potential conflict of interest.

Copyright (c) 2020 Tolaymat, Doré, Griffin, Shih, Edwards and Weiss. This is an open-access article distributed under the terms of the Creative Commons Attribution License (CC BY). The use, distribution or reproduction in other forums is permitted, provided the original author(s) and the copyright owner(s) are credited and that the original publication in this journal is cited, in accordance with accepted academic practice. No use, distribution or reproduction is permitted which does not comply with these terms. 